\title{
Joint distributions for stochastic functional differential equations*
}

\author{
ATSUSHI TAKEUCHI ${ }^{\dagger}$
}

\begin{abstract}
Consider stochastic functional differential equations, whose coefficients depend on past histories. The solution determines a non-Markov process. In the present paper, we shall obtain the existence of smooth densities for joint distributions of solutions, under the uniformly elliptic condition on the diffusion coefficients, via the Malliavin calculus. As an application, we shall study the computations of the Greeks on options associated with the asset price dynamics models with delayed effects.
\end{abstract}

Keywords: Stochastic functional differential equations, Malliavin calculus, Density function, Option pricing formula, Computations of the Greeks.

Mathematics Subject Classifications (2010): 34K50, 60H07, 62G07, 91G20, $91 \mathrm{G} 80$.

\section{Introduction}

Let $(\Omega, \mathscr{F}, \mathbb{P})$ be a probability space, and $r$ and $T$ be positive constants, which are fixed throughout the paper. Denote by $W=\left\{W(t)=\left(W^{1}(t), \ldots, W^{m}(t)\right) ; 0 \leq t \leq T\right\}$ the $m$-dimensional Brownian motion starting from the origin. Write $\mathscr{F}_{t}=\sigma[W(s) ; 0 \leq s \leq t] \vee \mathscr{N}$ for $0 \leq t<T$ and $\mathscr{F}_{T}=\mathscr{F}$, where $\mathscr{N}$ is the family of $\mathbb{P}$-null sets. Let $A_{0}, A_{1}, \ldots, A_{m}$ be in $C_{1+, b}^{1, \infty}\left([0, T] \times C\left([-r, 0] ; \mathbb{R}^{d}\right) ; \mathbb{R}^{d}\right)$, that is, those are jointly continuous in $(t, f) \in[0, T] \times C\left([-r, 0] ; \mathbb{R}^{d}\right)$ such that, for each $0 \leq i \leq m$,

- for each $f \in C\left([-r, 0] ; \mathbb{R}^{d}\right)$, the mapping $[0, T] \ni t \longmapsto A_{i}(t, f) \in \mathbb{R}^{d}$ is differentiable such that its partial derivative $\partial_{t} A_{i}(t, f)$ is bounded,

- for each $t \in[0, T]$, the mapping $C\left([-r, 0] ; \mathbb{R}^{d}\right) \ni f \longmapsto A_{i}(t, f) \in \mathbb{R}^{d}$ is smooth in the Fréchet sense such that all partial Fréchet derivatives $\nabla^{k} A_{i}(t, f)(k \in \mathbb{N})$ are bounded.

For a deterministic path $\eta \in C\left([-r, 0] ; \mathbb{R}^{d}\right)$, consider the stochastic functional differential equation:

$$
X(t)= \begin{cases}\eta(t) & (-r \leq t \leq 0), \\ \eta(0)+\int_{0}^{t} A_{0}\left(s, X_{s}\right) \mathrm{d} s+\int_{0}^{t} A\left(s, X_{s}\right) \mathrm{d} W(s) & (0 \leq t \leq T)\end{cases}
$$

To appear in Stochastics: An International Journal of Probability and Stochastic Processes

* Dedicated to Professor Takashi Komatsu on the occasion of his 70th birthday.

$\dagger$ E-mail address: takeuchi@sci.osaka-cu.ac.jp. Postal address: Department of Mathematics, Osaka City University, Sugimoto 3-3-138, Sumiyoshi-ku, Osaka 558-8585, Japan. 
where $A=\left(A_{1}, \ldots, A_{m}\right)$, and $X_{t}=\{X(t+u) ;-r \leq u \leq 0\}$ is the segment. Since the coefficients $A_{0}, A_{1}, \ldots, A_{m}$ satisfy the Lipschitz condition and the linear growth one, there exists a unique solution to (11) such that

$$
\mathbb{E}\left[\sup _{-r \leq t \leq T}|X(t)|^{p}\right] \leq C_{1, p, \eta, T}
$$

for any $p>1$. Moreover, the solution process $X=\{X(t) ;-r \leq t \leq T\}$ is non-Markovian, because the current state of the process depends on the whole past histories of the process $X$. See [8, 14, 15]. Thus, we cannot use any methods in analysis, partial differential equations and potential theory at all.

Such equation was initiated by Itô and Nisio [8] more than 50 years ago. It seems us to be very natural to study the models described by (1) stated above, because the models with their past histories often appear in finance, physics, biology and industry, etc. One of the typical examples in mathematical finance is the delayed Black-Scholes model studied in [1, 4, 5, 6, 13], which will be mentioned in Section 5. On the other hand, the Malliavin calculus is applicable to the study on the densities for the solution to (11). Kusuoka and Stroock [12] obtained the result on the existence of the smooth density for the solution with respect to the Lebesgue measure under the uniformly elliptic condition on the diffusion coefficients $A_{1}, \ldots, A_{m}$. Bell and Mohammed in [2, 3] also studied the same problem in case of stochastic delay differential equations such that $A_{i}(t, f)=\hat{A}_{i}(t, f(-r))(i=1 \ldots, m)$ for $t \in[0, T]$ and $f \in C\left([-r, 0]: \mathbb{R}^{d}\right)$, where $\hat{A}_{i}:[0, T] \times \mathbb{R}^{d} \rightarrow \mathbb{R}^{d}$ with some conditions on the boundedness and the regularity. They obtained in [2, 3] the existence of the smooth density under the degeneracy condition on $\sum_{i=1}^{m} \hat{A}_{i} \hat{A}_{i}^{*}$ by using the delay structure of the equation and conditioning the past history of the process, which is weaker than the uniformly elliptic condition on $\sum_{i=1}^{m} A_{i} A_{i}^{*}$. Furthermore, Kitagawa and Takeuchi [10] studied the asymptotic behavior of the density such as the Varadhan-type estimate for diffusion processes, by the large deviation theory and the Malliavin calculus, in which the constant $r$, called the delay parameter, plays a crucial role.

In the present paper, we will study the finite-dimensional joint distribution on the solution process to (1), from the viewpoint of the Malliavin calculus. As stated in Theorem 1 below, the joint distribution of the solution admits a smooth density under the uniformly elliptic condition on the diffusion coefficients $A_{1}, \ldots, A_{m}$. As an application, we shall also study the sensitivity analysis on the solution with respect to the initial state, which can be regarded as the computation of the Greeks for the options on the delayed asset price dynamics model.

The paper is organized as follows: Section 2 is devoted to a brief introduction of the Malliavin calculus and its application to stochastic functional differential equations. The result on the existence of the smooth density for the finite-dimensional joint distribution associated with the solution will be stated in Section 3. Sections 4 and 5 are typical applications of our result. In Section 4 we will study the sensitivity of the discrete and integral average related to the solution. The key points are to give the estimates on the Malliavin covariance matrices, which are implied by the uniformly elliptic conditions on the diffusion coefficients $A_{1}, \ldots, A_{m}$ of (1). We will study a delayed Black-Scholes model raised in [1, 13] in Section 5, in order to compute the Greeks on the options. 


\section{Malliavin calculus}

In this section, we shall apply the Malliavin calculus to the stochastic functional differential equation 11. See [16] on details of the Malliavin calculus. Let $\left(\mathbb{W}_{0}^{m}, \mathscr{W}, \mathbb{P}^{m}\right)$ be the Wiener space, that is, $\mathbb{W}_{0}^{m}$ is the set of $\mathbb{R}^{m}$-valued continuous functions on $[0, T]$ starting from the origin in $\mathbb{R}^{m}, \mathscr{W}$ is the topological $\sigma$-algebra on $\mathbb{W}_{0}^{m}$, and $\mathbb{P}^{W}$ is the Wiener measure over $\left(\mathbb{W}_{0}^{m}, \mathscr{W}\right)$. Denote by $\mathbb{H}_{0}^{m}$ be the Cameron-Martin subspace of $\mathbb{W}_{0}^{m}$ with the inner product

$$
\langle g, h\rangle_{\mathbb{H}_{0}^{m}}:=\int_{0}^{T}\langle\dot{g}(u), \dot{h}(u)\rangle_{\mathbb{R}^{m}} \mathrm{~d} u \quad\left(g, h \in \mathbb{H}_{0}^{m}\right),
$$

where $\dot{g}(u)$ is the derivative of $g$ in $u$.

For $0 \leq s \leq T$, let $\{Z(t, s) ;-r \leq t \leq T\}$ be the $\mathbb{R}^{d} \otimes \mathbb{R}^{d}$-valued process determined by

$$
Z(t, s)=\left\{\begin{array}{lr}
0 & (-r \leq t \leq 0 \text { or } t<s), \\
I_{d}+\int_{s}^{t} \nabla A_{0}\left(u, X_{u}\right) Z_{u}(\cdot, s) \mathrm{d} u+\int_{s}^{t} \sum_{i=1}^{m} \nabla A_{i}\left(u, X_{u}\right) Z_{u}(\cdot, s) \mathrm{d} W^{i}(u) & (s \leq t \leq T),
\end{array}\right.
$$

where $I_{d} \in \mathbb{R}^{d} \otimes \mathbb{R}^{d}$ is the identity, and $Z_{u}(\cdot, s)=\{Z(u+\tau, s) ;-r \leq \tau \leq 0\}$. Then, we have

Proposition 1 (cf. [10, 12]) For $-r \leq t \leq T$, the random variable $X(t)$ is smooth in the Malliavin sense. Moreover, the Malliavin derivative $D X(t)=\left\{D_{u} X(t) ; 0 \leq u \leq T\right\}$ of $X(t)$ and the Malliavin covariance matrix $V(t):=\langle D X(t), D X(t)\rangle_{\mathbb{H}_{0}^{m}}$ for $X(t)$ can be computed as follows:

$$
\begin{gathered}
D_{u} X(t)=\int_{0}^{u \wedge t} Z(t, s) A\left(s, X_{s}\right) d s \\
V(t)=\int_{0}^{t} \sum_{i=1}^{m} Z(t, s) A_{i}\left(s, X_{s}\right)\left\{Z(t, s) A_{i}\left(s, X_{s}\right)\right\}^{*} d s .
\end{gathered}
$$

Proof. The Malliavin smoothness of $X(t)$ can be justified by the limiting argument via the successive approximation $X^{(n)}=\left\{X^{(n)}(t) ;-r \leq t \leq T\right\}(n \in \mathbb{N} \cup\{0\})$ of the process $X$ :

$$
\begin{aligned}
X^{(0)}(t) & =\eta(t) \mathbb{I}_{[-r, 0]}(t)+\eta(0) \mathbb{I}_{(0, T]}(t), \\
X^{(n+1)}(t) & = \begin{cases}\eta(t) & (-r \leq t \leq 0), \\
\eta(0)+\int_{0}^{t} A_{0}\left(s, X_{s}^{(n)}\right) \mathrm{d} s+\int_{0}^{t} A\left(s, X_{s}^{(n)}\right) \mathrm{d} W(s) & (0<t \leq T)\end{cases}
\end{aligned}
$$

for $n \in \mathbb{N} \cup\{0\}$, and the inductive argument on the order of the derivatives. On the other hand, since $D_{u} X^{(n)}(t)=\mathbf{0}$ for $-r \leq t \leq 0$ and $n \in \mathbb{N} \cup\{0\}$, and

$$
\begin{aligned}
& D_{u} X^{(0)}(t)=0 \\
& \begin{aligned}
D_{u} X^{(n)}(t)= & \int_{0}^{u \wedge t} A\left(s, X_{s}^{(n-1)}\right) \mathrm{d} s+\int_{0}^{t} \nabla A_{0}\left(s, X_{s}^{(n-1)}\right) D_{u} X_{s}^{(n-1)} \mathrm{d} s \\
& +\int_{0}^{t} \sum_{i=1}^{m} \nabla A_{i}\left(s, X_{s}^{(n-1)}\right) D_{u} X_{s}^{(n-1)} \mathrm{d} W^{i}(s)
\end{aligned}
\end{aligned}
$$


for $n \in \mathbb{N}$ and $0 \leq t \leq T$, the limiting argument leads us to see that

$$
D_{u} X(t)=\left\{\begin{array}{lr}
0 & (-r \leq t \leq 0), \\
\int_{0}^{u \wedge t} A\left(s, X_{s}\right) \mathrm{d} s+\int_{0}^{t} \nabla A_{0}\left(s, X_{s}\right) D_{u} X_{s} \mathrm{~d} s+\int_{0}^{t} \sum_{i=1}^{m} \nabla A_{i}\left(s, X_{s}\right) D_{u} X_{s} \mathrm{~d} W^{i}(s) & (u \leq t \leq T) .
\end{array}\right.
$$

Thus, we can derive (3), because of the uniqueness of the solution to the equation (5). Moreover, the Malliavin covariance matrix $V(t)$ can be computed directly as (4).

\section{Density of joint distributions}

In this section, we shall mention the main result of the present paper. Before doing that, recall the classical result on the existence of the smooth density for the probability law of $X(t)$.

Lemma 1 (cf. [10, 12]) Suppose that the coefficients $A_{1}, \ldots, A_{m}$ satisfy the uniformly elliptic condition: there exists a constant $C_{2}>0$ such that

$$
\inf _{0 \leq t \leq T} \inf _{f \in C\left([-r, 0] ; \mathbb{R}^{d}\right)} \inf _{v \in \mathbb{S}^{d-1}} \sum_{i=1}^{m}\left(A_{i}(t, f) \cdot v\right)^{2} \geq C_{2} .
$$

Then, for each $0<t \leq T$, the probability law of the $\mathbb{R}^{d}$-valued random variable $X(t)$ admits a smooth density with respect to the Lebesgue measure on $\mathbb{R}^{d}$.

Let $n \in \mathbb{N}$ be arbitrary, and $0<t_{1}<\cdots<t_{n-1}<t_{n}=t$. Before introducing our result, we shall give an easy example, which is our motivation of our interests in the present paper.

Example 1 Consider the case of $m=d, A_{0}(t, f) \equiv 0$, and $A(t, f)=I_{d}$. Let $t_{0}=0$, and write

$$
p(t, x, y)=\frac{1}{\sqrt{2 \pi t}} \exp \left(-\frac{(y-x)^{2}}{2 t}\right) .
$$

Then, the process $X$ is the $d$-dimensional Brownian motion. Since

$$
\mathbb{P}\left[W\left(t_{1}\right) \in K_{1}, \ldots, W\left(t_{n}\right) \in K_{n}\right]=\int_{K_{1} \times \cdots \times K_{n}} p\left(t_{1}, 0, y_{1}\right) \prod_{k=2}^{n} p\left(t_{k}-t_{k-1}, y_{k-1}, y_{k}\right) \mathrm{d} y_{1} \cdots \mathrm{d} y_{n}
$$

for $K_{1}, \ldots, K_{n} \in \mathscr{B}\left(\mathbb{R}^{d}\right)$, the joint distribution $\left(W\left(t_{1}\right), \ldots, W\left(t_{n}\right)\right)$ is absolutely continuous with respect to the Lebesgue measure over $\mathbb{R}^{n d}$ such that its density function

$$
\varphi_{t_{1}, \ldots, t_{n}}\left(y_{1}, \ldots, y_{n}\right):=p\left(t_{1}, 0, y_{1}\right) \prod_{k=2}^{n} p\left(t_{k}-t_{k-1}, y_{k-1}, y_{k}\right)
$$

is smooth in $\left(y_{1}, \ldots, y_{n}\right) \in \mathbb{R}^{n d}$.

Now, we shall introduce the result in this paper, which can be regarded as the natural extension of Lemma1. 
Theorem 1 Under the condition (6) in Lemma 1 the joint distribution of $\left(X\left(t_{1}\right), \ldots, X\left(t_{n}\right)\right)$ admits a smooth density with respect to the Lebesgue masure on $\mathbb{R}^{n d}$.

Proof. The $\mathbb{R}^{n d}$-valued random variable $\left(X\left(t_{1}\right), \ldots, X\left(t_{n}\right)\right)$ determined by the equation (1) is smooth in the Malliavin sense, as stated in Proposition 1 because so are all of $X\left(t_{k}\right)(1 \leq k \leq n)$. Moreover, the corresponding Malliavin covariance matrix $V\left(t_{1}, \ldots, t_{n}\right)$ for $\left(X\left(t_{1}\right), \ldots, X\left(t_{n}\right)\right)$ is

$$
\begin{aligned}
V\left(t_{1}, \ldots, t_{n}\right) & =\left(\begin{array}{ccc}
\left\langle D X\left(t_{1}\right), D X\left(t_{1}\right)\right\rangle_{\mathbb{H}_{0}^{m}} & \cdots & \left\langle D X\left(t_{1}\right), D X\left(t_{n}\right)\right\rangle_{\mathbb{H}_{0}^{m}} \\
\vdots & \ddots & \vdots \\
\left\langle D X\left(t_{n}\right), D X\left(t_{1}\right)\right\rangle_{\mathbb{H}_{0}^{m}} & \cdots & \left\langle D X\left(t_{n}\right), D X\left(t_{n}\right)\right\rangle_{\mathbb{H}_{0}^{m}}
\end{array}\right) \\
& =\left(\begin{array}{cccc}
\int_{0}^{T} \Phi\left(t_{1}, s\right) \Phi\left(t_{1}, s\right)^{*} \mathrm{~d} s & \cdots & \int_{0}^{T} \Phi\left(t_{1}, s\right) \Phi\left(t_{n}, s\right)^{*} \mathrm{~d} s \\
\vdots & \ddots & \vdots \\
\int_{0}^{T} \Phi\left(t_{n}, s\right) \Phi\left(t_{1}, s\right)^{*} \mathrm{~d} s & \cdots & \int_{0}^{T} \Phi\left(t_{n}, s\right) \Phi\left(t_{n}, s\right)^{*} \mathrm{~d} s
\end{array}\right)
\end{aligned}
$$

where $\Phi(t, s):=Z(t, s) A\left(s, X_{s}\right) \mathbb{I}_{(s \leq t)}$ is $\mathbb{R}^{n d} \otimes \mathbb{R}^{n d}$-valued.

All we have to do is to study the negative-order moment of $\operatorname{det} V\left(t_{1}, \ldots, t_{n}\right)$. Let $v=\left(v_{1}, \ldots, v_{n}\right) \in \mathbb{R}^{n d}$ such that $|v|=\left(\left|v_{1}\right|^{2}+\cdots+\left|v_{n}\right|^{2}\right)^{1 / 2}=1$, and write $t_{0}=0$. Then, we have

$$
\begin{aligned}
\left\langle v, V\left(t_{1}, \ldots, t_{n}\right) v\right\rangle_{\mathbb{R}^{n d}}= & \int_{0}^{t_{n}}\left|\sum_{k=1}^{n} \Phi\left(t_{k}, s\right)^{*} v_{k}\right|^{2} \mathrm{~d} s \\
= & \sum_{j=1}^{n} \int_{t_{j-1}}^{t_{j}}\left|\sum_{k=j}^{n} \Phi\left(t_{k}, s\right)^{*} v_{k}\right|^{2} \mathrm{~d} s \\
\geq & \sum_{j=1}^{n-1} \int_{t_{j-1}}^{t_{j}}\left|\sum_{k=j}^{n} \Phi\left(t_{k}, s\right)^{*} v_{k}\right|^{2} \mathrm{~d} s \mathbb{I}_{\left(v_{n}=0\right)}+\int_{t_{n-1}}^{t_{n}}\left|\Phi\left(t_{n}, s\right)^{*} v_{n}\right|^{2} \mathrm{~d} s \mathbb{I}_{\left(v_{n} \neq 0\right)} \\
\geq & \sum_{j=1}^{n-2} \int_{t_{j-1}}^{t_{j}}\left|\sum_{k=j}^{n} \Phi\left(t_{k}, s\right)^{*} v_{k}\right|^{2} \mathrm{~d} s \mathbb{I}_{\left(v_{n-1}=0, v_{n}=0\right)} \\
& +\int_{t_{n-2}}^{t_{n-1}}\left|\Phi\left(t_{n-1}, s\right)^{*} v_{n-1}\right|^{2} \mathrm{~d} s \mathbb{I}_{\left(v_{n-1} \neq 0, v_{n}=0\right)}+\int_{t_{n-1}}^{t_{n}}\left|\Phi\left(t_{n}, s\right)^{*} v_{n}\right|^{2} \mathrm{~d} s \mathbb{I}_{\left(v_{n} \neq 0\right)} \\
\geq & \ldots \\
\geq & \sum_{j=1}^{n-1} \int_{t_{j-1}}^{t_{j}}\left|\Phi\left(t_{j}, s\right)^{*} v_{j}\right|^{2} \mathrm{~d} s \mathbb{I}_{\left(v_{j} \neq 0, v_{j+1}=0, \ldots, v_{n}=0\right)}+\int_{t_{n-1}}^{t_{n}}\left|\Phi\left(t_{n}, s\right)^{*} v_{n}\right|^{2} \mathrm{~d} s \mathbb{I}_{\left(v_{n} \neq 0\right)}
\end{aligned}
$$

Remark that

$$
\begin{aligned}
I_{j} & :=\int_{t_{j-1}}^{t_{j}}\left|\Phi\left(t_{j}, s\right)^{*} v_{j}\right|^{2} \mathrm{~d} s \mathbb{I}_{\left(v_{j} \neq 0, v_{j+1}=0, \ldots, v_{n}=0\right)} \\
& =\int_{t_{j-1}}^{t_{j}} \sum_{i=1}^{m}\left\langle Z\left(t_{j}, s\right) A_{i}\left(s, X_{s}\right), v_{j}\right\rangle_{\mathbb{R}^{d}}^{2} \mathrm{~d} s \mathbb{I}_{\left(v_{j} \neq 0, v_{j+1}=0, \ldots, v_{n}=0\right)}
\end{aligned}
$$




$$
\begin{aligned}
& \geq \int_{t_{j}-\lambda-\alpha}^{t_{j}} \sum_{i=1}^{m}\left\langle Z\left(t_{j}, s\right) A_{i}\left(s, X_{s}\right), v_{j}\right\rangle_{\mathbb{R}^{d}}^{2} \mathrm{~d} s \mathbb{I}_{\left(v_{j} \neq 0, v_{j+1}=0, \ldots, v_{n}=0\right)} \\
& \geq \frac{1}{2} \int_{t_{j}-\lambda-\alpha}^{t_{j}} \sum_{i=1}^{m}\left\langle A_{i}\left(s, X_{s}\right), v_{j}\right\rangle_{\mathbb{R}^{d}}^{2} \mathrm{~d} s \mathbb{I}_{\left(v_{j} \neq 0, v_{j+1}=0, \ldots, v_{n}=0\right)}-\int_{t_{j}-\lambda-\alpha}^{t_{j}} \sum_{i=1}^{m}\left|A_{i}\left(s, X_{s}\right)\right|^{2}\left|v_{j}\right|^{2}\left\|Z\left(t_{j}, s\right)-I_{d}\right\|^{2} \mathrm{~d} s \\
& \geq \frac{C_{2} \lambda^{-\alpha}}{2}\left|v_{j}\right|^{2} \mathbb{I}_{\left(v_{j} \neq 0, v_{j+1}=0, \ldots, v_{n}=0\right)}-C_{3}^{2} \lambda^{-\beta}
\end{aligned}
$$

on the subset

$$
\Omega_{2}:=\bigcap_{j=1}^{n}\left(\left\{\sup _{t_{j}-\lambda^{-\alpha} \leq s \leq t_{j}}|X(s)| \leq C_{3}\right\} \cap\left\{\int_{t_{j}-\lambda^{-\alpha}}^{t_{j}}\left\|Z\left(t_{j}, s\right)-I_{d}\right\|^{2} \mathrm{~d} s \leq \lambda^{-\beta}\right\}\right)
$$

under the condition (6) on $A_{1}, \ldots, A_{m}$, where $0<\alpha<1$ and $\alpha<\beta<2 \alpha$. Hence, it holds that

$$
\left\langle v, V\left(t_{1}, \ldots, t_{n}\right) v\right\rangle_{\mathbb{R}^{n d}} \geq \frac{C_{2} \lambda^{-\alpha}}{2}\left(\sum_{j=1}^{n-1}\left|v_{j}\right|^{2} \mathbb{I}_{\left(v_{j} \neq 0, v_{j+1}=0, \ldots, v_{n}=0\right)}+\left|v_{j}\right|^{2} \mathbb{I}_{\left(v_{n} \neq 0\right)}\right)-n C_{3}^{2} \lambda^{-\beta}
$$

on $\Omega_{2}$. On the other hand, we shall remark that

$$
\mathbb{P}\left[\Omega_{2}^{c}\right] \leq n\left(C_{3}^{-p} C_{1, p, \eta, T} \lambda^{-\alpha p / 2}+C_{4, p, T} \lambda^{-(2 \alpha-\beta) p}\right)
$$

for any $p>1$ from the Chebyshev inequality.

Now, we shall return to study the upper estimate of

$$
I(\lambda):=\sup _{v=\left(v_{1}, \ldots, v_{n}\right) \in \mathbb{R}^{n d},|v|=1} \mathbb{E}\left[\exp \left(-\lambda\left\langle v, V\left(t_{1}, \ldots, t_{n}\right) v\right\rangle_{\mathbb{R}^{n d}}\right)\right] .
$$

Since the mapping $\mathbb{S}^{n d-1} \ni v \longmapsto \mathbb{E}\left[\exp \left(-\lambda\left\langle v, V\left(t_{1}, \ldots, t_{n}\right) v\right\rangle_{\mathbb{R}^{n d}}\right)\right] \in \mathbb{R}$ is continuous, we can find

$$
\tilde{v}=\operatorname{argmax}\left\{\mathbb{E}\left[\exp \left(-\lambda\left\langle v, V\left(t_{1}, \ldots, t_{n}\right) v\right\rangle_{\mathbb{R}^{n d}}\right)\right] ; v=\left(v_{1}, \ldots, v_{n}\right) \in \mathbb{R}^{n d},|v|=1\right\} .
$$

Therefore, we can conclude that

$$
\begin{aligned}
I(\lambda) \equiv & \mathbb{E}\left[\exp \left(-\lambda\left\langle\tilde{v}, V\left(t_{1}, \ldots, t_{n}\right) \tilde{v}\right\rangle_{\mathbb{R}^{n d}}\right)\right] \\
\leq & \exp \left[-\lambda\left(\frac{C_{2} \lambda^{-\alpha}}{2}\left(\sum_{j=1}^{n-1}\left|\tilde{v}_{j}\right|^{2} \mathbb{I}_{\left(\tilde{v}_{j} \neq 0, \tilde{v}_{j+1}=0, \ldots, \tilde{v}_{n}=0\right)}+\left|\tilde{v}_{j}\right|^{2} \mathbb{I}_{\left(\tilde{v}_{n} \neq 0\right)}\right)-n C_{3}^{2} \lambda^{-\beta}\right)\right] \\
& \quad+n\left(C_{3}^{-p} C_{1, p, \eta, T} \lambda^{-\alpha p / 2}+C_{4, p, T} \lambda^{-(2 \alpha-\beta) p}\right) \\
= & o\left(\lambda^{-C_{5} p}\right)
\end{aligned}
$$

as $\lambda \rightarrow+\infty$ for any $p>1$, so we can obtain

$$
\begin{aligned}
\mathbb{E}\left[\left(\operatorname{det} V\left(t_{1}, \ldots, t_{n}\right)\right)^{-q}\right] & =\mathbb{E}\left[\left(\inf _{v \in \mathbb{S}^{n d-1}}\left\langle v, V\left(t_{1}, \ldots, t_{n}\right) v\right\rangle_{\mathbb{R}^{n d}}\right)^{-n q d}\right] \\
& \leq C_{6} \sup _{v \in \mathbb{S}^{n d-1}} \mathbb{E}\left[\left(\left\langle v, V\left(t_{1}, \ldots, t_{n}\right) v\right\rangle_{\mathbb{R}^{n d}}\right)^{-(n q d+4 n d-4)}\right]+C_{7} \\
& =\frac{C_{6}}{\Gamma(n q d+4 n d-4)} \int_{0}^{+\infty} \lambda^{4 q d+4 n d-5} I(\lambda) \mathrm{d} \lambda+C_{7}<+\infty
\end{aligned}
$$

for any $q>1$. See [11] on the detailed discussion of the second inequality stated above. 
Remark 1 Bell and Mohammed [3] have studied the stochastic delay equation with hereditary drift:

$$
X(t)= \begin{cases}\eta(t) & (-r \leq t \leq 0), \\ \eta(0)+\int_{0}^{t} \hat{A}_{0}(s,\{X(u) ;-r \leq u \leq s\}) \mathrm{d} s+\int_{0}^{t} \hat{A}(s, X(s-r)) \mathrm{d} W(s) & (0 \leq t \leq T),\end{cases}
$$

where $\hat{A}_{0}:[0, T] \times C\left([-r, T] ; \mathbb{R}^{d}\right)$ such that $\hat{A}_{0}(t, f)\left(t \in[0, T], f \in C\left([-r, T] ; \mathbb{R}^{d}\right)\right)$ depends only on $\{f(s) ;-r \leq s \leq t\}$, and $\hat{A}_{i}:[0, T] \times \mathbb{R}^{d} \rightarrow \mathbb{R}^{d}(i=1, \ldots, m)$ with the certain conditions on the boundedness and the regularity. Write $\hat{A}=\left(\hat{A}_{1}, \ldots, \hat{A}_{m}\right)$, and let $t \in[0, T]$. They showed in [3] the existence of the smooth density for the law of $X(t)$, under the degeneracy of the $\mathbb{R}^{d} \otimes \mathbb{R}^{d}$-valued function $\hat{A} \hat{A}^{*}$ of polynomial order on hypersurfaces in $\mathbb{R}^{d}$, which is weaker than the condition (6) in the present paper, by using the delay structure in (7) and conditioning on the past history of the process.

As for the equation (7), we can also derive the same assertions as Lemma 1 and Theorem 1 , under the degeneracy condition on $\hat{A} \hat{A}^{*}$ as stated in [3]. In fact, since it can be checked, similarly to Proposition 11 that the Malliavin calculus is applicable to the solution of the equation (7), our goal in the argument is to study the negative-order moment of the determinants on the corresponding Malliavin covariance matrices for $X(t)$ and $\left(X\left(t_{1}\right), \ldots, X\left(t_{n}\right)\right)$ of the forms:

$$
\begin{aligned}
V(t) & =\int_{0}^{t} D_{u} X(t)\left(D_{u} X(t)\right)^{*} \mathrm{~d} u \\
V\left(t_{1}, \ldots, t_{n}\right) & =\left(\begin{array}{ccc}
\int_{0}^{t_{n}} D_{u} X\left(t_{1}\right)\left(D_{u} X\left(t_{1}\right)\right)^{*} \mathrm{~d} u & \cdots & \int_{0}^{t_{n}} D_{u} X\left(t_{1}\right)\left(D_{u} X\left(t_{n}\right)\right)^{*} \mathrm{~d} u \\
\vdots & \ddots & \vdots \\
\int_{0}^{t_{n}} D_{u} X\left(t_{n}\right)\left(D_{u} X\left(t_{1}\right)\right)^{*} \mathrm{~d} u & \cdots & \int_{0}^{t_{n}} D_{u} X\left(t_{n}\right)\left(D_{u} X\left(t_{n}\right)\right)^{*} \mathrm{~d} u
\end{array}\right),
\end{aligned}
$$

where $0=t_{0}<t_{1}<\cdots<t_{n-1}<t_{n}=t$ such that $\|\Delta\|:=\max _{1 \leq j \leq n}\left(t_{j}-t_{j-1}\right) \leq r$. Then, we have only to check the lower estimates of the quadratic forms of $V(t)$ and $V\left(t_{1}, \ldots, t_{n}\right)$ :

$$
\begin{gathered}
\langle v, V(t) v\rangle_{\mathbb{R}^{d}}=\int_{0}^{t}\left|\left(D_{u} X(t)\right)^{*} v\right|^{2} \mathrm{~d} u \\
\left\langle\left(v_{1}, \ldots, v_{n}\right), V\left(t_{1}, \ldots, t_{n}\right)\left(v_{1}, \ldots, v_{n}\right)\right\rangle_{\mathbb{R}^{n d}} \geq \\
\sum_{j=1}^{n-1} \int_{t_{j-1}}^{t_{j}}\left|\left(D_{u} X\left(t_{j}\right)\right)^{*} v_{j}\right|^{2} \mathrm{~d} u \mathbb{I}_{\left(v_{j} \neq 0, v_{j+1}=0, \ldots, v_{n}=0\right)} \\
+\int_{t_{n-1}}^{t_{n}}\left|\left(D_{u} X\left(t_{n}\right)\right)^{*} v_{n}\right|^{2} \mathrm{~d} u \mathbb{I}_{\left(v_{n} \neq 0\right)},
\end{gathered}
$$

where $v \in \mathbb{R}^{d}$ with $|v|=1$, and $\left(v_{1}, \ldots, v_{n}\right) \in \mathbb{R}^{n d}$ with $\sum_{j=1}^{n}\left|v_{j}\right|^{2}=1$.

Finally, we shall remark that it would be open whether Lemma 1 and Theorem 1 on the equation (11) can be obtained, under the degeneracy condition as stated in [3], because the special forms of the diffusion coefficients $\hat{A}_{1}, \ldots, \hat{A}_{m}$ in the equation (7) play a crucial role in [3],

\section{Applications}

In this section, we shall study one of the typical applications of Theorem 1. Consider the case of $d=$ $m=1$ throughout this section, in order to avoid the complication of our argument. Let $f \in C^{1}(\mathbb{R} ; \mathbb{R})$ 
such that there exist positive constants $C_{8}$ and $C_{9, f}$ satisfying with

$$
\inf _{|x| \leq C_{8}}\left|f^{\prime}(x)\right|^{2} \geq C_{9, f}
$$

Then, we can find the inverse function of $f$ around the origin. Define

$$
Y(t):=\frac{1}{t} \int_{0}^{t} f(X(s)) \mathrm{d} s \quad(t \in(0, T]) .
$$

Theorem 2 Suppose the uniformly elliptic condition (6) on $A_{1}$ stated in Lemma $\square$ Then, for each $0<$ $t \leq T$, the probability law of $Y(t)$ admits a smooth density with respect to the Lebesgue measure on $\mathbb{R}$.

Proof. We have only to check the negative-order integrability of the Malliavin covariance $\tilde{V}(t)$ of $Y(t)$, because the Malliavin smoothness of $Y(t)$ can be derived by the one of $X(s)$ for each $0 \leq s \leq t$. See [16].

Firstly, we shall compute the Malliavin covariance $\tilde{V}(t):=\langle D Y(t), D Y(t)\rangle_{\mathbb{H}_{0}^{1}}$. Since

$$
\begin{aligned}
\frac{\mathrm{d}}{\mathrm{d} u} D_{u} Y(t) & =\frac{1}{t} \int_{0}^{t} f^{\prime}(X(s)) \frac{\mathrm{d}}{\mathrm{d} u} D_{u} X(s) \mathrm{d} s \\
& =\frac{1}{t} \int_{u}^{t} f^{\prime}(X(s)) Z(s, u) A_{1}\left(u, X_{u}\right) \mathrm{d} s,
\end{aligned}
$$

for $0 \leq u \leq t$, the Malliavin covariance $\tilde{V}(t)$ of $Y(t)$ can be computed as follows:

$$
\begin{aligned}
\tilde{V}(t) & =\int_{0}^{t}\left(\frac{\mathrm{d}}{\mathrm{d} u} D_{u} Y(t)\right)^{2} \mathrm{~d} u \\
& =\int_{0}^{t}\left\{\frac{1}{t}\left(\int_{u}^{t} f^{\prime}(X(s)) Z(s, u) \mathrm{d} s\right) A_{1}\left(u, X_{u}\right)\right\}^{2} \mathrm{~d} u .
\end{aligned}
$$

Secondly, we shall check the negative-order integrability of $\tilde{V}(t)$. In order to do it, it is sufficient to observe $\mathbb{E}\left[\exp (-\lambda \tilde{V}(t)]=o\left(\lambda^{-p}\right)\right.$ as $\lambda \rightarrow+\infty$ for any $p>1$, because

$$
\mathbb{E}\left[\tilde{V}(t)^{-p}\right]=\frac{1}{\Gamma(p)} \int_{0}^{+\infty} \lambda^{p-1} \mathbb{E}[\exp (-\lambda \tilde{V}(t))] \mathrm{d} \lambda .
$$

Let $0<\gamma<1 / 3$ be a constant, and $\lambda>1$ sufficiently large. Write $t_{\lambda}:=t-\lambda^{-\gamma}$ and

$$
\Omega_{3}:=\left\{\sup _{t_{\lambda} \leq s \leq t}|X(s)| \leq C_{8}\right\} \cap\left\{\sup _{t_{\lambda} \leq s \leq t}\left|Z\left(s, \theta\left(t_{\lambda}, t\right)\right)-1\right|^{2} \leq \frac{1}{4}\right\} .
$$

We shall remark that

$$
\begin{aligned}
\mathbb{P}\left[\Omega_{3}^{c}\right] & \leq \mathbb{P}\left[\sup _{t_{\lambda} \leq s \leq t}|X(s)|>C_{8}\right]+\mathbb{P}\left[\sup _{t_{\lambda} \leq s \leq t}\left|Z\left(s, \theta\left(t_{\lambda}, t\right)\right)-1\right|^{2}>\frac{1}{4}\right] \\
& \leq C_{8}^{-p} \mathbb{E}\left[\sup _{t_{\lambda} \leq s \leq t}|X(s)|^{p}\right]+4^{p} \mathbb{E}\left[\sup _{t_{\lambda} \leq s \leq t}\left|Z\left(s, \theta\left(t_{\lambda}, t\right)\right)-1\right|^{2 p}\right] \\
& \leq C_{1, p, \eta, T} C_{8}^{-p} \lambda^{-\gamma p / 2}+C_{4, p, T} 4^{p} \lambda^{-\gamma p}
\end{aligned}
$$


from the Chebyshev inequality. Moreover, the mean value theorem tells us to see that

$$
\begin{aligned}
\tilde{V}(t) \geq & \frac{1}{t^{2}} \int_{t_{\lambda}}^{t}\left\{\left(\int_{u}^{t} f^{\prime}(X(s)) Z(s, u) \mathrm{d} s\right) A_{1}\left(u, X_{u}\right)\right\}^{2} \mathrm{~d} u \\
= & \left.\frac{(1-\theta)^{2}}{t^{2}} \lambda^{-3 \gamma}\left\{f^{\prime}(X(s)) Z(s, u) A_{1}\left(u, X_{u}\right)\right\}^{2}\right|_{u=\theta\left(t_{\lambda}, t\right), s=\delta(u, t)} \\
\geq & \frac{(1-\theta)^{2}}{t^{2}} \lambda^{-3 \gamma}\left(\inf _{|x| \leq C_{11}}\left|f^{\prime}(x)\right|^{2}\right) \\
& \quad \times\left(\inf _{0 \leq u \leq T} \inf _{g \in C([-r, 0] ; \mathbb{R})}\left|A_{1}(u, g)\right|^{2}\right) Z\left(\delta\left(\theta\left(t_{\lambda}, t\right), t\right), \theta\left(t_{\lambda}, t\right)\right)^{2} \\
\geq & \frac{(1-\theta)^{2}}{t^{2}} \lambda^{-3 \gamma} C_{9, f} C_{2}\left(\frac{1}{2}-\sup _{\theta\left(t_{\lambda}, t\right) \leq s \leq t}\left|Z\left(s, \theta\left(t_{\lambda}, t\right)\right)-1\right|^{2}\right) \\
\geq & \frac{(1-\theta)^{2}}{4 t^{2}} C_{9, f} C_{2} \lambda^{-3 \gamma}
\end{aligned}
$$

on $\Omega_{3}$, where $0<\theta<1$ and $0<\delta<1$ are constants, $\theta\left(t_{\lambda}, t\right):=\theta t+(1-\theta) t_{\lambda}$, and $\delta(u, t):=\delta t+(1-$ $\delta) u$. In the fifth inequality, we have used the assumption on the function $f$, and the uniformly elliptic condition (6) on $A_{1}$. Write $\hat{\mathbb{E}}[\cdot]=\mathbb{E}\left[\cdot \mathbb{I}_{\Omega_{3}}\right]$. Then, we can get

$$
\begin{aligned}
\mathbb{E}[\exp (-\lambda \tilde{V}(t))] & \leq \hat{\mathbb{E}}[\exp (-\lambda \tilde{V}(t))]+\mathbb{P}\left[\Omega_{3}^{c}\right] \\
& \leq \exp \left[-\frac{(1-\theta)^{2}}{4 t^{2}} C_{9, f} C_{2} \lambda^{1-3 \gamma}\right]+C_{1, p, \eta, T} C_{8}^{-p} \lambda^{-\gamma p / 2}+C_{4, p, T} 4^{p} \lambda^{-\gamma p} \\
& =o\left(\lambda^{-C_{10} p}\right)
\end{aligned}
$$

as $\lambda \rightarrow+\infty$ for any $p>1$, which is our desired conclusion.

Remark 2 We can also obtain Theorem 2 from the viewpoint of Theorem 1 Let $0=t_{0}<t_{1}<\cdots<t_{N}=t$ such that $\|\Delta\|:=\max _{1 \leq k \leq N}\left(t_{k}-t_{k-1}\right)$ tends to 0 as $N \rightarrow+\infty$. Write

$$
Y(t)=\frac{1}{t} \int_{0}^{t} f(X(s)) \mathrm{d} s, \quad Y_{N}\left(t_{1}, \ldots, t_{N}\right):=\frac{1}{N} \sum_{k=1}^{N} f\left(X\left(t_{k}\right)\right) .
$$

The corresponding Malliavin covariances to $Y(t)$ and $Y_{N}\left(t_{1}, \ldots, t_{N}\right)$ are

$$
\begin{aligned}
& \tilde{V}(t)=\int_{0}^{T}\left\{\frac{1}{t} \int_{0}^{t} f^{\prime}(X(s)) Z(s, u) A_{1}\left(u, X_{u}\right) \mathbb{I}_{(u \leq s)} \mathrm{d} s\right\}^{2} \mathrm{~d} u, \\
& \tilde{V}_{N}\left(t_{1}, \ldots, t_{N}\right)=\int_{0}^{T}\left\{\frac{1}{N} \sum_{k=1}^{N} f^{\prime}\left(X\left(t_{k}\right)\right) Z\left(t_{k}, u\right) A_{1}\left(u, X_{u}\right) \mathbb{I}_{\left(u \leq t_{k}\right)}\right\}^{2} \mathrm{~d} u .
\end{aligned}
$$

As pointed out in the proof of Theorem 2 the Malliavin smoothness of $Y(t)$ and $Y_{N}\left(t_{1}, \ldots, t_{N}\right)$ can be checked directly from the one of $X(s)$ for $0 \leq s \leq t$. On the other hand, in order to study the negative-order moment of $\tilde{V}_{N}(t)$, it is sufficient to give the one ov $\tilde{V}_{N}\left(t_{1}, \ldots, t_{N}\right)$, because the dominated convergence theorem and the Fatou lemma lead us to see that

$$
\mathbb{E}\left[\tilde{V}(t)^{-q}\right]=\frac{1}{\Gamma(q)} \int_{0}^{+\infty} \lambda^{q-1} \mathbb{E}[\exp (-\lambda \tilde{V}(t))] \mathrm{d} \lambda
$$




$$
\begin{aligned}
& =\frac{1}{\Gamma(q)} \int_{0}^{+\infty} \lambda^{q-1} \lim _{N \rightarrow+\infty} \mathbb{E}\left[\exp \left(-\lambda \tilde{V}_{N}\left(t_{1}, \ldots, t_{N}\right)\right)\right] \mathrm{d} \lambda \\
& \leq \liminf _{N \rightarrow+\infty} \frac{1}{\Gamma(q)} \int_{0}^{+\infty} \lambda^{q-1} \mathbb{E}\left[\exp \left(-\lambda \tilde{V}_{N}\left(t_{1}, \ldots, t_{N}\right)\right)\right] \mathrm{d} \lambda \\
& \leq \liminf _{N \rightarrow+\infty} \mathbb{E}\left[\tilde{V}_{N}\left(t_{1}, \ldots, t_{N}\right)^{-q}\right] .
\end{aligned}
$$

Now, we shall study the estimate of $\mathbb{E}\left[\tilde{V}_{N}\left(t_{1}, \ldots, t_{N}\right)^{-q}\right]$. Let $q>1$ be arbitrary and $0<\sigma<1 / 3$ a constant. Write $N_{\sigma, t}:=(N / t)^{1 / \sigma}$. Remark that

$$
\begin{aligned}
\mathbb{E}\left[\tilde{V}_{N}\left(t_{1}, \ldots, t_{N}\right)^{-q}\right]= & \frac{1}{\Gamma(q)} \int_{0}^{+\infty} \lambda^{q-1} \mathbb{E}\left[\exp \left(-\lambda \tilde{V}_{N}\left(t_{1}, \ldots, t_{N}\right)\right)\right] \mathrm{d} \lambda \\
\leq & \frac{1}{\Gamma(q)} \int_{0}^{1} \lambda^{q-1} \mathrm{~d} \lambda+\frac{1}{\Gamma(q)} \int_{1}^{N_{\sigma, t}} \lambda^{q-1} \mathbb{E}\left[\exp \left(-\lambda \tilde{V}_{N}\left(t_{1}, \ldots, t_{N}\right)\right)\right] \mathrm{d} \lambda \\
& \quad+\frac{1}{\Gamma(q)} \int_{N_{\sigma, t}}^{+\infty} \lambda^{q-1} \mathbb{E}\left[\exp \left(-\lambda \tilde{V}_{N}\left(t_{1}, \ldots, t_{N}\right)\right)\right] \mathrm{d} \lambda \\
=: & I_{1, N}+I_{2, N}+I_{3, N} .
\end{aligned}
$$

First of all, the estimate of $I_{1, N}$ is trivial:

$$
I_{1, N} \equiv \frac{1}{\Gamma(q)} \int_{0}^{1} \lambda^{q-1} \mathrm{~d} \lambda=\frac{1}{\Gamma(q+1)}<+\infty .
$$

Secondly, we shall consider the estimate of $I_{2, N}$. Since $Z(t, u)=0$ for $t<u$, the mean value theorem implies that

$$
\begin{aligned}
\tilde{V}_{N}\left(t_{1}, \ldots, t_{N}\right) & =\int_{0}^{t}\left\{\frac{1}{N} \sum_{k=1}^{N} f^{\prime}\left(X\left(t_{k}\right)\right) Z\left(t_{k}, u\right) A_{1}\left(u, X_{u}\right) \mathbb{I}_{\left(u \leq t_{k}\right)}\right\}^{2} \mathrm{~d} u \\
& \geq \int_{t_{N-1}}^{t}\left\{\frac{1}{N} \sum_{k=1}^{N} f^{\prime}\left(X\left(t_{k}\right)\right) Z\left(t_{k}, u\right) A_{1}\left(u, X_{u}\right) \mathbb{I}_{\left(u \leq t_{k}\right)}\right\}^{2} \mathrm{~d} u \\
& =\int_{t_{N-1}}^{t}\left\{\frac{1}{N} f^{\prime}(X(t)) Z(t, u) A_{1}\left(u, X_{u}\right)\right\}^{2} \mathrm{~d} u \\
& =\left\{\frac{1}{N} f^{\prime}(X(t)) Z\left(t, \theta_{2}\left(t_{N-1}, t\right)\right) A_{1}\left(\theta_{2}\left(t_{N-1}, t\right), X_{\theta_{2}\left(t_{N-1}, t\right)}\right)\right\}^{2} N_{\sigma, t}^{-\sigma},
\end{aligned}
$$

where $0<\theta_{2}<1$ is a constant, and $\theta_{2}(s, t):=s+\theta_{2}(t-s)$. Denote by

$$
\Omega_{4}:=\left\{\sup _{t_{N-1} \leq s \leq t}|X(s)| \leq C_{8}\right\} \cap\left\{\sup _{\theta_{2}\left(t_{N-1}, t\right) \leq s \leq t}\left|Z\left(s, \theta_{2}\left(t_{N-1}, t\right)\right)-1\right|^{2} \leq \frac{1}{4}\right\} .
$$

We shall remark that

$$
\mathbb{P}\left[\Omega_{4}^{c}\right] \leq \mathbb{P}\left[\sup _{t_{N-1} \leq s \leq t}|X(s)|>C_{8}\right]+\mathbb{P}\left[\sup _{\theta_{2}\left(t_{N-1}, t\right) \leq s \leq t}\left|Z\left(s, \theta_{2}\left(t_{N-1}, t\right)\right)-1\right|^{2}>\frac{1}{4}\right]
$$




$$
\begin{aligned}
& \leq C_{8}^{-p} \mathbb{E}\left[\sup _{t_{N-1} \leq s \leq t}|X(s)|^{p}\right]+4^{p} \mathbb{E}\left[\sup _{\theta_{2}\left(t_{N-1}, t\right) \leq s \leq t}\left|Z\left(s, \theta_{2}\left(t_{N-1}, t\right)\right)-1\right|^{2 p}\right] \\
& \leq C_{1, p, \eta, T} C_{8}^{-p} N_{\sigma, t}^{-\sigma p / 2}+C_{4, p, T} 4^{p} N_{\sigma, t}^{-\sigma p}
\end{aligned}
$$

from the Chebyshev inequality. Moreover, we see that, on $\Omega_{4}$,

$$
\begin{aligned}
\tilde{V}_{N}\left(t_{1}, \ldots, t_{N}\right) & \geq\left\{\frac{1}{N} f^{\prime}(X(t)) Z\left(t, \theta_{2}\left(t_{N-1}, t\right)\right) A_{1}\left(\theta_{2}\left(t_{N-1}, t\right), X_{\theta_{2}\left(t_{N-1}, t\right)}\right)\right\}^{2} N_{\sigma, t}^{-\sigma} \\
& \geq \frac{C_{9, f} C_{2} N_{\sigma, t}^{-\sigma}}{N^{2}} Z\left(s, \theta_{2}\left(t_{N-1}, t\right)\right)^{2} \\
& \geq \frac{C_{9, f} C_{2} N_{\sigma, t}^{-\sigma}}{4 N^{2}}
\end{aligned}
$$

from the assumption on the function $f$ and the condition (6). Write $\hat{E}[\cdot]:=\mathbb{E}\left[\cdot \mathbb{I}_{\Omega_{4}}\right]$. Hence, it holds that

$$
\begin{aligned}
\mathbb{E}\left[\exp \left(-\lambda \tilde{V}_{N}\left(t_{1}, \ldots, t_{N}\right)\right)\right] & \leq \hat{\mathbb{E}}\left[\exp \left(-\lambda \tilde{V}_{N}\left(t_{1}, \ldots, t_{N}\right)\right)\right]+\mathbb{P}\left[\Omega_{4}^{c}\right] \\
& \leq \exp \left(-\frac{C_{9, f} C_{2} N_{\sigma, t}^{-\sigma}}{4 N^{2}} \lambda\right)+C_{1, p, \eta, T} C_{8}^{-p} N_{\sigma, t}^{-\sigma p / 2}+C_{4, p, T} 4^{p} N_{\sigma, t}^{-\sigma p} \\
& \leq \exp \left(-\frac{C_{9, f} C_{2} N_{\sigma, t}^{-\sigma}}{4 N^{2}} \lambda\right)+\left(C_{1, p, \eta, T} C_{8}^{-p}+C_{4, p, T} 4^{p}\right) N_{\sigma, t}^{-\sigma p / 2}
\end{aligned}
$$

Let $p>(2 q / \sigma) \vee 1$ be arbitrary. Thus, the mean value theorem enables us to get

$$
\begin{aligned}
I_{2, N} \equiv & \frac{1}{\Gamma(q)} \int_{1}^{N_{\sigma, t}} \lambda^{q-1} \mathbb{E}\left[\exp \left(-\lambda \tilde{V}_{N}\left(t_{1}, \ldots, t_{N}\right)\right)\right] \mathrm{d} \lambda \\
\leq & \frac{1}{\Gamma(q)} \int_{1}^{N_{\sigma, t}} \lambda^{q-1}\left\{\exp \left(-\frac{C_{9, f} C_{2} N_{\sigma, t}^{-\sigma}}{4 N^{2}} \lambda\right)+\left(C_{1, p, \eta, T} C_{8}^{-p}+C_{4, p, T} 4^{p}\right) N_{\sigma, t}^{-\sigma p / 2}\right\} \mathrm{d} \lambda \\
\leq & \frac{\lambda^{q-1}}{\Gamma(q)} \exp \left(-\frac{\left.C_{9, f} C_{2} N_{\sigma, t}^{-\sigma} \lambda\right)\left.\right|_{\lambda=\left(1-\delta_{2}\right)+\delta_{2} N_{\sigma, t}}\left(N_{\sigma, t}-1\right)}{4 N^{2}} \lambda\right. \\
& +\frac{1}{\Gamma(q)} \int_{1}^{N_{\sigma, t}}\left(C_{1, p, \eta, T} C_{8}^{-p}+C_{4, p, T} 4^{p}\right) \lambda^{q-\sigma p / 2-1} \mathrm{~d} \lambda \\
\leq & \frac{C_{11, q}}{\Gamma(q)}\left(1+N_{\sigma, t}^{q}\right) \exp \left[-\frac{C_{9, f} C_{2} \delta_{2} N_{\sigma, t}^{1-\sigma}}{4 N^{2}}\right] \\
& +\frac{1}{\Gamma(q)} \int_{1}^{+\infty}\left(C_{1, p, \eta, T} C_{8}^{-p}+C_{4, p, T} 4^{p}\right) \lambda^{q-\sigma p / 2-1} \mathrm{~d} \lambda \\
= & \frac{C_{11, q}}{\Gamma(q)}\left(1+N_{\sigma, t}^{q}\right) \exp \left[-\frac{C_{9, f} C_{2} \delta_{2} N_{\sigma, t}^{1-\sigma}}{4 N^{2}}\right]+\frac{C_{1, p, \eta, T} C_{9}^{-p}+C_{4, p, T} 4^{p}}{(\sigma p / 2-q) \Gamma(q)} \\
\rightarrow & \frac{C_{1, p, \eta, T} C_{8}^{-p}+C_{4, p, T} 4^{p}}{(\sigma p / 2-q) \Gamma(q)} \quad(N \rightarrow+\infty),
\end{aligned}
$$

because of $0<\sigma<1 / 3$, where $0<\delta_{2}<1$ is a constant. 
Thirdly, we shall consider the estimate of $I_{3, N}$. Write $t_{\lambda}:=t-\lambda^{-\sigma}$, and let $\lambda \geq N_{\sigma, t}$ be sufficiently large. The mean value theorem implies that

$$
\begin{aligned}
\tilde{V}_{N}\left(t_{1}, \ldots, t_{N}\right) & =\int_{0}^{t}\left\{\frac{1}{N} \sum_{k=1}^{N} f^{\prime}\left(X\left(t_{k}\right)\right) Z\left(t_{k}, u\right) A_{1}\left(u, X_{u}\right) \mathbb{I}_{\left(u \leq t_{k}\right)}\right\}^{2} \mathrm{~d} u \\
& \geq \int_{t_{\lambda}}^{t}\left\{\frac{1}{N} \sum_{k=1}^{N} f^{\prime}\left(X\left(t_{k}\right)\right) Z\left(t_{k}, u\right) A_{1}\left(u, X_{u}\right) \mathbb{I}_{\left(u \leq t_{k}\right)}\right\}^{2} \mathrm{~d} u \\
& =\int_{t_{\lambda}}^{t}\left\{\frac{1}{N} f^{\prime}(X(t)) Z(t, u) A_{1}\left(u, X_{u}\right)\right\}^{2} \mathrm{~d} u \\
& =\left\{\frac{1}{N} f^{\prime}(X(t)) Z\left(t, \theta_{3}\left(t_{\lambda}, t\right)\right) A_{1}\left(\theta_{3}\left(t_{\lambda}, t\right), X_{\theta_{3}\left(t_{\lambda}, t\right)}\right)\right\}^{2} \lambda^{-\sigma},
\end{aligned}
$$

where $0<\theta_{3}<1$ is a constant, and $\theta_{3}(s, t):=s+\theta_{3}(t-s)$. Denote by

$$
\Omega_{5, \lambda}:=\left\{\sup _{t_{\lambda} \leq s \leq t}|X(s)| \leq C_{8}\right\} \cap\left\{\sup _{\theta_{3}\left(t_{\lambda}, t\right) \leq s \leq t}\left|Z\left(s, \theta_{3}\left(t_{\lambda}, t\right)\right)-1\right|^{2} \leq \frac{1}{4}\right\} .
$$

Then, we can get

$$
\begin{aligned}
\tilde{V}_{N}\left(t_{1}, \ldots, t_{N}\right) & \geq\left\{\frac{1}{N} f^{\prime}(X(t)) Z\left(t, \theta_{3}\left(t_{\lambda}, t\right)\right) A_{1}\left(\theta_{3}\left(t_{\lambda}, t\right), X_{\theta_{3}\left(t_{\lambda}, t\right)}\right)\right\}^{2} \lambda^{-\sigma} \\
& \geq \frac{C_{9, f} C_{2}}{N^{2}} Z\left(s, \theta_{3}\left(t_{\lambda}, t\right)\right)^{2} \lambda^{-\sigma} \\
& \geq \frac{C_{9, f} C_{2}}{4 t^{2}} \lambda^{-3 \sigma}
\end{aligned}
$$

on $\Omega_{5, \lambda}$, from the assumption on the function $f$ and the uniformly elliptic condition (6), because of $t / N=N_{\sigma, t}^{-\sigma} \geq \lambda^{-\sigma}$. Define $\hat{\mathbb{E}}_{\lambda}[\cdot]:=\mathbb{E}\left[\cdot \mathbb{I}_{\Omega_{5, \lambda}}\right]$. Then, we have

$$
\begin{aligned}
\mathbb{E}\left[\exp \left(-\lambda \tilde{V}_{N}\left(t_{1}, \ldots, t_{N}\right)\right)\right] & \leq \hat{\mathbb{E}}_{\lambda}\left[\exp \left(-\lambda \tilde{V}_{N}\left(t_{1}, \ldots, t_{N}\right)\right)\right]+\mathbb{P}\left[\Omega_{5, \lambda}^{c}\right] \\
& \leq \exp \left(-\frac{C_{9, f} C_{2}}{4 t^{2}} \lambda^{1-3 \sigma}\right)+C_{1, p, \eta, T} C_{8}^{-p} \lambda^{-\sigma p / 2}+C_{4, p, T} 4^{p} \lambda^{-\sigma p} \\
& \leq \exp \left(-\frac{C_{9, f} C_{2}}{4 t^{2}} \lambda^{1-3 \sigma}\right)+\left(C_{1, p, \eta, T} C_{8}^{-p}+C_{4, p, T} 4^{p}\right) \lambda^{-\sigma p / 2}
\end{aligned}
$$

from the Chebyshev inequality. Hence, it holds that

$$
\begin{aligned}
I_{3, N} & \equiv \frac{1}{\Gamma(q)} \int_{t_{\sigma, N}}^{+\infty} \lambda^{q-1} \mathbb{E}\left[\exp \left(-\lambda \tilde{V}_{N}\left(t_{1}, \ldots, t_{N}\right)\right)\right] \mathrm{d} \lambda \\
& \leq \frac{1}{\Gamma(q)} \int_{t_{\sigma, N}}^{+\infty} \lambda^{q-1}\left\{\exp \left(-\frac{C_{9, f} C_{2}}{4 t^{2}} \lambda^{1-3 \sigma}\right)+\left(C_{1, p, \eta, T} C_{8}^{-p}+C_{4, p, T} 4^{p}\right) \lambda^{-\sigma p / 2}\right\} \mathrm{d} \lambda \\
& \leq \frac{1}{\Gamma(q)} \int_{1}^{+\infty} \lambda^{q-1}\left\{\exp \left(-\frac{C_{9, f} C_{2}}{4 t^{2}} \lambda^{1-3 \sigma}\right)+\left(C_{1, p, \eta, T} C_{8}^{-p}+C_{4, p, T} 4^{p}\right) \lambda^{-\sigma p / 2}\right\} \mathrm{d} \lambda \\
& \leq \frac{\left(4 t^{2}\right)^{q /(1-3 \sigma)} \Gamma(q /(1-3 \sigma))}{(1-3 \sigma)\left(C_{9, f} C_{2}\right)^{q /(1-3 \sigma)} \Gamma(q)}+\frac{C_{1, p, \eta, T} C_{8}^{-p}+C_{4, p, T} 4^{p}}{(q-\sigma p / 2) \Gamma(q)} .
\end{aligned}
$$


Therefore, we see that

$$
\begin{aligned}
\mathbb{E}\left[\tilde{V}(t)^{-q}\right] \leq & \liminf _{N \rightarrow+\infty}\left(I_{1, N}+I_{2, N}+I_{3, N}\right) \\
\leq & \frac{1}{\Gamma(q+1)}+\frac{C_{1, p, \eta, T} C_{8}^{-p}+C_{4, p, T} 4^{p}}{(\sigma p / 2-q) \Gamma(q)} \\
& +\left\{\frac{\left(4 t^{2}\right)^{q /(1-3 \sigma)} \Gamma(q /(1-3 \sigma))}{(1-3 \sigma)\left(C_{9, f} C_{2}\right)^{q /(1-3 \sigma)} \Gamma(q)}+\frac{C_{1, p, \eta, T} C_{8}^{-p}+C_{4, p, T} 4^{p}}{(q-\alpha p / 2) \Gamma(q)}\right\}<+\infty,
\end{aligned}
$$

which is our desired one.

\section{Delayed Black-Scholes models and sensitivity analysis}

In this section, we shall apply our studies to the option pricing of the asset price dynamics model with delayed effects. See [1, 4, 5, 6, 13] on details. Let $A_{i} \in C_{0+, b}^{1}(\mathbb{R} ; \mathbb{R})(i=0,1)$ with the uniformly elliptic condition of the form: there exists a positive constant $C_{12}$ with

$$
\inf _{y \in \mathbb{R}} A_{1}(y)^{2} \geq C_{12}
$$

Let $x>0$ be a constant. Consider the $\mathbb{R}$-valued process $X=\{X(t) ;-r \leq t \leq T\}$ determined by the stochastic delay differential equation of the form:

$$
X(t)= \begin{cases}x & (-r \leq t \leq 0), \\ x+\int_{0}^{t} A_{0}(X(s-r)) X(s) \mathrm{d} s+\int_{0}^{t} A_{1}(X(s-r)) X(s) \mathrm{d} W(s) & (0<t \leq T) .\end{cases}
$$

The existence of the unique solution to (14) can be checked easily. In fact, it is trivial on the interval $[-r, 0]$. Since

$$
X(t)=x+\int_{0}^{t} A_{0}(x) X(s) \mathrm{d} s+\int_{0}^{t} A_{1}(x) X(s) \mathrm{d} W(s)
$$

for $0<t \leq r$, we can derive our conclusion from the Lipschitz condition and the linear growth condition on the coefficients. Iterating such argument enables us to get our desired assertion on each intervals $[k r,(k+1) r](k \in \mathbb{N} \cup\{0\})$. Moreover, the equation (14) can be solved as

$$
X(t)=\left\{\begin{array}{l}
x \\
x \exp \left[\int_{0}^{t} A_{1}(X(s-r)) \mathrm{d} W(s)+\int_{0}^{t}\left\{A_{0}(X(s-r))-\frac{A_{1}(X(s-r))^{2}}{2}\right\} \mathrm{d} s\right] \quad(0 \leq t \leq T),
\end{array}\right.
$$

which implies that $X(t)>0$ a.s. The process $X=\{X(t) ;-r \leq t \leq T\}$ is called the delayed Black-Scholes model in mathematical finance (cf. [1, 4, 5, 6, 13]).

Remark 3 Although the coefficients of the equation (14) do not satisfy the uniformly elliptic condition (6) as stated in Lemma 1, the probability law of $X(t)$ admits a smooth density with respect to 
the Lebesgue measure on $\mathbb{R}$. Denote by $\tilde{x}=\log x$ and $\tilde{A}_{i}(\tilde{y})=A_{i}\left(e^{\tilde{y}}\right)(i=0,1)$. Then, the process $\tilde{X}=\{\tilde{X}(t):=\log X(t) ;-r \leq t \leq T\}$ satisfies

$$
\tilde{X}(t)= \begin{cases}\tilde{x} & (-r \leq t \leq 0), \\ \tilde{x}+\int_{0}^{t} \tilde{A}_{1}(\tilde{X}(s-r)) \mathrm{d} W(s)+\int_{0}^{t}\left\{\tilde{A}_{0}(\tilde{X}(s-r))-\frac{A_{1}(\tilde{X}(s-r))}{2}\right\} \mathrm{d} s \quad(0 \leq t \leq T) .\end{cases}
$$

Since the coefficient $\tilde{A}_{1}$ satisfies

$$
\inf _{\tilde{y} \in \mathbb{R}} \tilde{A}_{1}(\tilde{y})^{2}=\inf _{\tilde{y} \in \mathbb{R}} A_{1}\left(e^{\tilde{y}}\right)^{2} \geq C_{12}
$$

from (13), we can conclude from Lemma 1 that the probability law of the $\mathbb{R}$-valued random variable $\tilde{X}(t)$ admits a smooth density $\tilde{p}_{t}(\tilde{y})$ with respect to the Lebesgue measure on $\mathbb{R}$. Hence, the density of the probability law of $X(t)=\exp (\tilde{X}(t))$ is

$$
p_{t}(y)=\frac{\tilde{p}_{t}(\log y)}{y} \quad(y>0)
$$

which is smooth in $y>0$.

Let $R>0$ be a constant, which denotes the rate of return of a riskless asset. Denote by $B=$ $\{B(t) ;-r \leq t \leq T\}$ the riskless asset price process, which is given by

$$
B(t)=\mathbb{I}_{[-r, 0]}(t)+e^{R t} \mathbb{I}_{(0, T]}(t) .
$$

Write $\bar{X}=\{\bar{X}(t):=X(t) / B(t) ;-r \leq t \leq T\}$, which is called the discounted stock price process. Then, the Itô formula leads us to see that, for $0<t \leq T$,

$$
\begin{aligned}
\mathrm{d} \bar{X}(t) & =\left\{A_{0}(X(t-r))-R\right\} \bar{X}(t) \mathrm{d} t+A_{1}(X(t-r)) \bar{X}(t) \mathrm{d} W(t) \\
& =A_{1}(X(t-r)) \bar{X}(t)\{\mathrm{d} W(t)-\Sigma(t) \mathrm{d} t\},
\end{aligned}
$$

where $\Sigma(t)=-\left\{A_{0}(X(t-r))-R\right\} / A_{1}(X(t-r))$. Define the process $M=\{M(t) ; 0 \leq t \leq T\}$ by

$$
M(t):=\exp \left[\int_{0}^{t} \Sigma(s) \mathrm{d} W(s)-\frac{1}{2} \int_{0}^{t} \Sigma(s)^{2} \mathrm{~d} s\right] .
$$

Then, the process $M$ is a square-integrable $\left(\mathscr{F}_{t}\right)$-martingale, because

$$
\mathbb{E}\left[M(t)^{2}\right] \leq \mathbb{E}\left[\exp \left\{8 \int_{0}^{t} \Sigma(s)^{2} \mathrm{~d} s\right\}\right]^{1 / 2}<+\infty
$$

from the boundedness of $A_{0}$ and the uniformly elliptic condition (13) on $A_{1}$. In particular, we have $\mathbb{E}[M(T)]=1$. Then, the measure $\mathrm{d} \mathbb{Q}:=M(T) \mathrm{d} \mathbb{P}$ is also the probability one on the measurable space $(\Omega, \mathscr{F})$, and the Girsanov theorem tells us to see that the process $\tilde{W}=\left\{\tilde{W}(t):=W(t)-\int_{0}^{t} \Sigma(s) \mathrm{d} s ; 0 \leq\right.$ $t \leq T\}$ is also a Brownian motion starting from the origin under the measure $\mathrm{d} \mathbb{Q}$. Let $Z$ be a $\mathscr{F}_{T}-$ measurable, non-negative and integrable random variable, which is called a contingent claim on the process $X=\{X(t) ;-r \leq t \leq T\}$. 
Proposition 2 The market $(X, B)=\{(X(t), B(t)) ; 0 \leq t \leq T\}$ is complete.

Proof. Remark that $\mathscr{F}_{t}^{\tilde{W}}=\mathscr{F}_{t}$. Since the process $L=\left\{L(t):=\mathbb{E}_{\mathbb{Q}}\left[e^{-R T} Z \mid \mathscr{F}_{t}\right] ; 0 \leq t \leq T\right\}$ is an $\left(\mathscr{F}_{t}\right)$ martingale under $\mathrm{d} \mathbb{Q}$, we can find an $\left(\mathscr{F}_{t}\right)$-predictable, square-integrable process $\varphi=\{\varphi(t) ; 0 \leq t \leq T\}$ such that

$$
L(t)=L(0)+\int_{0}^{t} \varphi(s) \mathrm{d} \tilde{W}(s)
$$

by the martingale representation theorem (cf. [7]-Theorem II-6.6, p.80). Set

$$
\pi_{X}(t):=\frac{\varphi(t)}{A_{1}(X(t-r)) \tilde{X}(t)}, \quad \pi_{B}(t):=L(t)-\pi_{X}(t) \tilde{X}(t), \quad V(t):=\pi_{B}(t) B(t)+\pi_{X}(t) X(t) .
$$

Then, since $V(t)=B(t) L(t)$, the Itô formula implies that

$$
\begin{aligned}
\mathrm{d} V(t) & =B(t) \mathrm{d} L(t)+L(t) \mathrm{d} B(t) \\
& =B(t) \varphi(t) \mathrm{d} \tilde{W}(t)+\left\{\pi_{B}(t)+\pi_{X}(t) \tilde{X}(t)\right\} \mathrm{d} B(t) \\
& =\pi_{X}(t)\left\{A_{1}(X(t-r)) X(t)(\mathrm{d} W(t)-\Sigma(t) \mathrm{d} t)+R X(t) \mathrm{d} t\right\}+\pi_{B}(t) \mathrm{d} B(t) \\
& =\pi_{X}(t) \mathrm{d} X(t)+\pi_{B}(t) \mathrm{d} B(t),
\end{aligned}
$$

which means that $\left(\pi_{X}, \pi_{B}\right)=\left\{\left(\pi_{X}(t), \pi_{B}(t)\right) ; 0 \leq t \leq T\right\}$ is a self-financing strategy. Moreover, since

$$
V(T)=e^{R T} L(T)=\mathbb{E}_{\mathbb{Q}}\left[Z \mid \mathscr{F}_{T}\right]=Z,
$$

the contingent claim $Z$ is attainable. Hence, the market $(X, B)=\{(X(t), B(t)) ; 0 \leq t \leq T\}$ is complete, which is our goal in the proposition.

Proposition 3 For $0 \leq t \leq T$, it holds that

$$
V(t)=e^{-R(T-t)} \mathbb{E}_{\mathbb{Q}}\left[Z \mid \mathscr{F}_{t}\right] .
$$

Proof. As seen in the proof of Proposition 2, it holds that

$$
\mathrm{d}\left(\frac{V(t)}{B(t)}\right)=\mathrm{d} L(t)=\varphi(t) \mathrm{d} \tilde{W}(t) .
$$

Hence, the discounted process $V / B=\{V(t) / B(t) ; 0 \leq t \leq T\}$ is an $\left(\mathscr{F}_{t}\right)$-martingale under $\mathrm{d} \mathbb{Q}$. Then, the fair price $V(t)$ of the claim $Z$ is given by

$$
\frac{V(t)}{B(t)}=\mathbb{E}_{\mathbb{Q}}\left[\frac{V(T)}{B(T)} \mid \mathscr{F}_{t}\right]=\mathbb{E}_{\mathbb{Q}}\left[e^{-R T} Z \mid \mathscr{F}_{t}\right]
$$

because of $V(T)=e^{R T} L(T)=\mathbb{E}_{\mathbb{Q}}\left[Z \mid \mathscr{F}_{T}\right]=Z$.

From now on, we shall consider the case $A_{0} \equiv 0$ only, because the Girsanov transform enables us to discuss the general case, if we want. For the sake of simplicity of notations, we shall note $\mathbb{P}$ and $W$ instead of $\mathbb{Q}$ and $\tilde{W}$. Since

$$
X(t)= \begin{cases}x & (-r \leq t \leq 0), \\ x+\int_{0}^{t} A_{1}(X(s-r)) X(s) \mathrm{d} W(s) & (t \in[0, T])\end{cases}
$$


we have

$$
\partial_{x} X(t)= \begin{cases}1 & (t \in[-r, 0]) \\ 1+\int_{0}^{t} A_{1}^{\prime}(X(s-r)) \partial_{x} X(s-r) X(s) \mathrm{d} W(s)+\int_{0}^{t} A_{1}(X(s-r)) \partial_{X} X(s) \mathrm{d} W(s) & (0 \leq t \leq T) .\end{cases}
$$

Let $U=\{U(t) ;-r \leq t \leq T\}$ and $\hat{U}=\{\hat{U}(t) ;-r \leq t \leq T\}$ be $\mathbb{R}$-valued processes determined by the equations:

$$
\begin{array}{lc}
U(t)=\hat{U}(t)=1 & (-r \leq t \leq 0), \\
U(t)=1+\int_{0}^{t} A_{1}(X(s-r)) U(s) \mathrm{d} W(s) & (0 \leq t \leq T), \\
\hat{U}(t)=1-\int_{0}^{t} \hat{U}(s) A_{1}(X(s-r)) \mathrm{d} W(s)+\int_{0}^{t} \hat{U}(s) A_{1}(X(s-r))^{2} \mathrm{~d} s & (0 \leq t \leq T) .
\end{array}
$$

Then, it is clear that $U(t) \hat{U}(t)=\hat{U}(t) U(t)=1$, via the Itô formula, and that

$$
\begin{aligned}
\partial_{x} X(t)= & U(t)\left\{1+\int_{0}^{t} \hat{U}(s) A_{1}^{\prime}(X(s-r)) \partial_{x} X(s-r) X(s) \mathrm{d} W(s)\right. \\
& \left.-\int_{0}^{t} \hat{U}(s) A_{1}(X(s-r)) A_{1}^{\prime}(X(s-r)) \partial_{x} X(s-r) \mathrm{d} s\right\} \\
= & : U(t) \Lambda(t) .
\end{aligned}
$$

Moreover, for $0 \vee(t-r) \leq u \leq t$, we see that $Z(t, u)=U(t) \hat{U}(u)$ is invertible, because

$$
\begin{aligned}
Z(t, u) & =1+\int_{u}^{t} A_{1}^{\prime}(X(s-r)) Z(s-r, u) X(s) \mathrm{d} W(s)+\int_{u}^{t} A_{1}(X(s-r)) Z(s, u) \mathrm{d} W(s) \\
& =1+\int_{u}^{t} A_{1}(X(s-r)) Z(s, u) \mathrm{d} W(s)
\end{aligned}
$$

for $0 \vee(t-r) \leq u \leq t$,

$$
U(t) \hat{U}(u)=1+\int_{u}^{t} A_{1}(X(s-r)) U(s) \hat{U}(u) \mathrm{d} W(s),
$$

and the uniqueness of the solutions.

Now, we shall state the result on the Greeks computation on the European option with respect to the initial point $x$. Denote by $C_{L G}(\mathbb{R} ; \mathbb{R})$ the set of continuous functions with the linear growth order, and define

$$
\mathfrak{F}(\mathbb{R} ; \mathbb{R})=\left\{\sum_{k=1}^{n} \alpha_{k} f_{k} \mathbb{I}_{K_{k}} ; n \in \mathbb{N}, \alpha_{k} \in \mathbb{R}, f_{k} \in C_{L G}(\mathbb{R} ; \mathbb{R}), K_{k} \subset \mathbb{R}: \text { interval }\right\} .
$$

Theorem 3 (Greeks computation of the European option) For $\Phi \in \mathfrak{F}(\mathbb{R}, ; \mathbb{R})$, it holds that

$$
\partial_{x} \mathbb{E}[\Phi(X(t))]=\mathbb{E}\left[\Phi(X(t)) \Gamma_{E}(t)\right]
$$


where

$$
\Gamma_{E}(t)=\frac{1}{t \wedge r} \delta\left(\frac{U(\cdot) \Lambda(t)}{A_{1}(X(\cdot-r)) X(\cdot)} \mathbb{I}_{[0 \vee(t-r), t]}(\cdot)\right),
$$

and $\delta(\cdot)$ is the Skorokhod integral operator.

Proof. Consider the case of $\Phi \in C_{b}^{1}(\mathbb{R} ; \mathbb{R})$. Choose $0 \vee(t-r) \leq u \leq t$. Since

$$
\begin{aligned}
\frac{\mathrm{d}}{\mathrm{d} u} D_{u}(\Phi(X(t))) & =\Phi^{\prime}(X(t)) \frac{\mathrm{d}}{\mathrm{d} u} D_{u} X(t) \\
& =\Phi^{\prime}(X(t)) Z(t, u) A_{1}(X(u-r)) X(u) \\
& =\Phi^{\prime}(X(t)) U(t) \hat{U}(u) A_{1}(X(u-r)) X(u) \\
& =\partial_{x}(\Phi(X(t))) \frac{\hat{U}(u) A_{1}(X(u-r)) X(u)}{\Lambda(t)}
\end{aligned}
$$

from the chain rule on the operator $D$, the integration by parts formula leads us to get

$$
\begin{aligned}
\partial_{x}(\mathbb{E}[\Phi(X(t))]) & =\mathbb{E}\left[\partial_{x}(\Phi(X(t)))\right] \\
& =\mathbb{E}\left[\frac{1}{t \wedge r} \int_{0 \vee(t-r)}^{t} \frac{\mathrm{d}}{\mathrm{d} u} D_{u}(\Phi(X(t))) \frac{U(u) \Lambda(t)}{A_{1}(X(u-r)) X(u)} \mathrm{d} u\right] \\
& =\mathbb{E}\left[\Phi(X(t)) \frac{1}{t \wedge r} \delta\left(\frac{U(\cdot) \Lambda(t)}{A_{1}(X(\cdot-r)) X(\cdot)} \mathbb{I}_{[0 \vee(t-r), t]}(\cdot)\right)\right] \\
& =\mathbb{E}\left[\Phi(X(t)) \Gamma_{E}(t)\right] .
\end{aligned}
$$

In order to extend the class of payoff functions, we have to find a sequence $\left\{\Phi_{n} ; n \in \mathbb{N}\right\}$ such that

$$
\begin{aligned}
& \sup _{x \in K}\left|\mathbb{E}\left[\Phi_{n}(X(t))\right]-\mathbb{E}[\Phi(X(t))]\right| \rightarrow 0, \\
& \sup _{x \in K}\left|\partial_{x} \mathbb{E}\left[\Phi_{n}(X(t))\right]-\mathbb{E}\left[\Phi(X(t)) \Gamma_{E}(t)\right]\right| \rightarrow 0
\end{aligned}
$$

as $n \rightarrow+\infty$, where $K$ is a compact subset in $[0,+\infty)$. Hence, it is sufficient to justify

$$
\mathbb{E}\left[\left|\Phi_{n}(X(t))-\Phi(X(t))\right|^{2}\right] \rightarrow 0
$$

as $n \rightarrow+\infty$. See [9, 17] on details.

As for $\Phi \in C_{b}(\mathbb{R} ; \mathbb{R})$, it is easy to find the sequence $\left\{\Phi_{n} ; n \in \mathbb{N}\right\}$ in $C_{b}^{1}(\mathbb{R} ; \mathbb{R})$ satisfying with (20). When $\Phi$ is the indicator function, we can approximate $\Phi$ by a sequence $\left\{\Phi_{n} ; n \in \mathbb{N}\right\}$ in $C_{b}([0,+\infty) ; \mathbb{R})$. In order to give the convergence (20), we need the result on the existence of the smooth density for $X(t)$, which have already stated in Remark 3, under the uniformly elliptic condition (13). Since a continuous function with linear growth order can be approximated by bounded continuous functions, we can extend to the class $\mathfrak{F}(\mathbb{R} ; \mathbb{R})$ easily. 
Remark 4 From Proposition 1.3 .5 (p.40) in [16], it holds that

$$
\begin{aligned}
& \delta\left(\frac{U(\cdot) \Lambda(t)}{A_{1}(X(\cdot-r)) X(\cdot)} \mathbb{I}_{[0 \vee(t-r), t]}(\cdot)\right) \\
& =\delta\left(\frac{U(\cdot)}{A_{1}(X(\cdot-r)) X(\cdot)} \mathbb{I}_{[0 \vee(t-r), t]}(\cdot)\right) \Lambda(t)-\int_{0 \vee(t-r)}^{t} \frac{U(u)}{A_{1}(X(u-r)) X(u)} \frac{\mathrm{d}}{\mathrm{d} u} D_{u} \Lambda(t) \mathrm{d} u \\
& =\left(\int_{0 \vee(t-r)}^{t} \frac{U(u)}{A_{1}(X(u-r)) X(u)} \mathrm{d} W(u)\right) \Lambda(t)-\int_{0 \vee(t-r)}^{t} \frac{U(u)}{A_{1}(X(u-r)) X(u)} \frac{\mathrm{d}}{\mathrm{d} u} D_{u} \Lambda(t) \mathrm{d} u .
\end{aligned}
$$

In particular, consider the case $0 \leq t \leq r$. Since

$$
U(u)=\frac{X(u)}{x}, \quad \Lambda(t)=1+x A_{1}^{\prime}(x) W(t)-x A_{1}^{\prime}(x) A_{1}(x) t
$$

for $0 \leq u \leq t$, we can get

$$
\delta\left(\frac{U(\cdot) \Lambda(t)}{A_{1}(X(\cdot-r)) X(\cdot)} \mathbb{I}_{[0 \vee(t-r), t]}(\cdot)\right)=\frac{W(t)}{x A_{1}(x)}+\frac{A_{1}^{\prime}(x)}{A_{1}(x)}\left(W(t)^{2}-t\right)-A_{1}(x) t W(t) .
$$

We shall compute the Delta, that is, a kind of the Greeks for the Asian-type option associated with an asset price model with delay in the initial point $x>0$. Write $\tilde{x}=\log x$. For $\tilde{y} \in \mathbb{R}$, define the $\mathbb{R}$-valued processes $\tilde{X}(t)=\{\tilde{X}(t) ;-r \leq t \leq T\}$ and $\tilde{Y}=\{\tilde{Y}(t) ;-r \leq t \leq T\}$ by

$$
\begin{aligned}
& \tilde{X}(t)= \begin{cases}\tilde{x} & (-r \leq t \leq 0), \\
\tilde{x}-\frac{1}{2} \int_{0}^{t} \tilde{A}_{1}(\tilde{X}(s-r))^{2} \mathrm{~d} s+\int_{0}^{t} \tilde{A}_{1}(\tilde{X}(s-r)) \mathrm{d} W(s) & (0 \leq t \leq T),\end{cases} \\
& \tilde{Y}(t)= \begin{cases}\tilde{y} & (-r \leq t \leq 0), \\
\tilde{y}+\int_{0}^{t} \exp (\tilde{X}(s)) \mathrm{d} s & (0 \leq t \leq T),\end{cases}
\end{aligned}
$$

where $\tilde{A}_{1}(\tilde{z})=A_{1}(\exp (z))$. Remark that $X(t)=\exp \{\tilde{X}(t)\}$ and $Y_{t}=\tilde{Y}(t) / t$. Consider the $\mathbb{R}^{2}$-valued process $\mathfrak{X}=\{\mathfrak{X}(t)=(\tilde{X}(t), \tilde{Y}(t)) ;-r \leq t \leq T\}$ given by $\mathfrak{X}(t)=(\tilde{x}, \tilde{y})^{*}=: \tilde{\mathfrak{X}}$ for $-r \leq t \leq 0$, and

$$
\begin{aligned}
\mathfrak{X}(t) & =\left(\begin{array}{c}
\tilde{x} \\
\tilde{y}
\end{array}\right)+\int_{0}^{t}\left(\begin{array}{c}
-\tilde{A}_{1}(\tilde{X}(s-r))^{2} / 2 \\
\exp (\tilde{X}(s))
\end{array}\right) \mathrm{d} s+\int_{0}^{t}\left(\begin{array}{c}
\tilde{A}_{1}(\tilde{X}(s-r)) \\
0
\end{array}\right) \mathrm{d} W(s) \\
& =: \tilde{\mathfrak{X}}+\int_{0}^{t} \mathfrak{A}_{0}(\mathfrak{X}(s-r), \mathfrak{X}(s)) \mathrm{d} s+\int_{0}^{t} \mathfrak{A}_{1}(\mathfrak{X}(s-r)) \mathrm{d} W(s)
\end{aligned}
$$

for $0 \leq t \leq T$. Let $\pi: \mathbb{R}^{2} \rightarrow \mathbb{R}$ be the canonical projection defined by $\pi(\mathfrak{X})=y$ for $\mathfrak{X}=(x, y)^{*} \in \mathbb{R}^{2}$. Remark that our main interest is to study the sensitivity of

$$
\mathbb{E}[\Phi(\tilde{Y}(t) / t)]=\mathbb{E}[(\Phi \circ \pi)(\mathfrak{X}(t) / t)]
$$


for a certain payoff function $\Phi$. Before introducing our result, we shall prepare some notations. For $0 \leq u \leq T$, let $\mathfrak{Z}(\cdot, u)=\{\mathfrak{Z}(t, u) ;-r \leq t \leq T\}$ be the $\mathbb{R}^{2} \otimes \mathbb{R}^{2}$-valued process determined by the equation

$$
\mathfrak{Z}(t, u)=\left\{\begin{array}{cc}
0 & (-r \leq t \leq 0 \text { or } t<u), \\
I_{2}+\int_{u}^{t} \partial_{1} \mathfrak{A}_{1}(\mathfrak{X}(s-r)) \mathfrak{Z}(s-r, u) \mathrm{d} W(s) & \\
\quad+\int_{u}^{t}\left\{\partial_{1} \mathfrak{A}_{0}(\mathfrak{X}(s-r), \mathfrak{X}(s)) \mathfrak{Z}(s-r, u)\right. & \\
& \left.+\partial_{2} \mathfrak{A}_{0}(\mathfrak{X}(s-r), \mathfrak{X}(s)) \mathfrak{Z}(s, u)\right\} \mathrm{d} s \quad(u \leq t \leq T),
\end{array}\right.
$$

where $I_{2} \in \mathbb{R}^{2} \otimes \mathbb{R}^{2}$ is the identity.

Theorem 4 (Greeks computation of the Asian option) For $\Phi \in \mathfrak{F}(\mathbb{R}, ; \mathbb{R})$, it holds that

$$
\partial_{x} \mathbb{E}[\Phi(\tilde{Y}(t) / t)]=\mathbb{E}\left[\Phi(\tilde{Y}(t) / t) \Gamma_{A}(t)\right]
$$

where $\mathfrak{V}(t)$ is the Malliavin covariance matrix for $\mathfrak{X}(t)$, and

$$
\Gamma_{A}(t)=\frac{1}{x} \delta\left(\left(\mathfrak{Z}(t, \cdot) \mathfrak{A}_{1}(\mathfrak{X}(\cdot-r))\right)^{*} \mathfrak{V}(t)^{-1} \partial_{\mathfrak{X}} \mathfrak{X}(t)\right)(1,0)^{*} .
$$

Proof. Similarly to Theorem 3, the proof is based upon the standard density argument as seen in [9, 17]. We shall remark that, as for the case that $\Phi$ is the indicator function, the most crucial point is the existence of the smooth density for $Y(t)$ as stated in Theorem 2

We have only to discuss the case of $\Phi \in C_{b}^{1}(\mathbb{R} ; \mathbb{R})$. First of all, we shall remark that

$$
\partial_{\tilde{x}} \mathbb{E}[\Phi(\tilde{Y}(t) / t)]=\partial_{\mathfrak{X}} \mathbb{E}[(\Phi \circ \pi)(\mathfrak{X}(t) / t)](1,0)^{*} .
$$

We can compute the Malliavin derivative of $\mathfrak{X}(t)$ as follows:

$$
\frac{\mathrm{d}}{\mathrm{d} u} D_{u} \mathfrak{X}(t)=\mathfrak{Z}(t, u) \mathfrak{A}_{1}(\mathfrak{X}(u-r)) \mathbb{I}_{[0, t]}(u),
$$

so, the Malliavin covariance matrix $\mathfrak{V}(t):=\langle D \mathfrak{X}(t), D \mathfrak{X}(t)\rangle_{\mathbb{H}_{0}^{1}}$ for $\mathfrak{X}(t)$ is

$$
\mathfrak{V}(t)=\int_{0}^{t} \mathfrak{Z}(t, u) \mathfrak{A}_{1}(\mathfrak{X}(u-r))\left(\mathfrak{Z}(t, u) \mathfrak{A}_{1}(\mathfrak{X}(u-r))\right)^{*} \mathrm{~d} u
$$

Suppose that the inverse of $\operatorname{det} \mathfrak{V}(t)$ is in $\mathbb{L}^{p}(\Omega)$ for any $p>1$. Since

$$
\begin{aligned}
\partial_{\tilde{\mathfrak{X}}}\{(\Phi \circ \pi)(\mathfrak{X}(t))\} & =\partial(\Phi \circ \pi)(\mathfrak{X}(t)) \partial_{\tilde{\mathfrak{X}}} \mathfrak{X}(t) \\
& =\int_{0}^{t} \frac{\mathrm{d}}{\mathrm{d} u} D_{u}\{(\Phi \circ \pi)(\mathfrak{X}(t))\}\left(\mathfrak{Z}(t, u) \mathfrak{A}_{1}(\mathfrak{X}(u-r))\right)^{*} \mathrm{~d} u \mathfrak{V}(t)^{-1} \partial_{\tilde{\mathfrak{X}}} \mathfrak{X}(t),
\end{aligned}
$$

the integration by parts formula leads us to see that

$$
\partial_{\tilde{x}} \mathbb{E}[\Phi(\tilde{Y}(t) / t)]=\partial_{\tilde{X}} \mathbb{E}[(\Phi \circ \pi)(\mathfrak{X}(t) / t)](1,0)^{*}
$$




$$
\begin{aligned}
& =\mathbb{E}\left[\partial(\Phi \circ \pi)(\mathfrak{X}(t) / t) \frac{\partial_{\tilde{X}} \mathfrak{X}(t)}{t}\right](1,0)^{*} \\
& =\mathbb{E}\left[\int_{0}^{t} \frac{\mathrm{d}}{\mathrm{d} u} D_{u}\{(\Phi \circ \pi)(\mathfrak{X}(t) / t)\}\left(\mathfrak{Z}(t, u) \mathfrak{A}_{1}(\mathfrak{X}(u-r))\right)^{*} \mathrm{~d} u \mathfrak{V}(t)^{-1} \partial_{\tilde{X}} \mathfrak{X}(t)\right](1,0)^{*} \\
& =\mathbb{E}\left[(\Phi \circ \pi)(\mathfrak{X}(t) / t) \delta\left(\left(\mathfrak{Z}(t, \cdot) \mathfrak{A}_{1}(\mathfrak{X}(\cdot-r))\right)^{*} \mathfrak{V}(t)^{-1} \partial_{\tilde{X}} \mathfrak{X}(t)\right)\right](1,0)^{*} \\
& =\mathbb{E}\left[\Phi(\tilde{Y}(t) / t) \delta\left(\left(\mathfrak{Z}(t, \cdot) \mathfrak{A}_{1}(\mathfrak{X}(\cdot-r))\right)^{*} \mathfrak{V}(t)^{-1} \partial_{\tilde{\mathfrak{X}}} \mathfrak{X}(t)\right)\right](1,0)^{*} .
\end{aligned}
$$

Remark that

$$
\partial_{\mathfrak{X}} \mathfrak{X}(t)= \begin{cases}I_{2} & (-r \leq t \leq 0), \\ I_{2}+\int_{0}^{t} \partial_{1} \mathfrak{A}_{1}(\mathfrak{X}(s-r)) \partial_{\mathfrak{X}} \mathfrak{X}(s-r) \mathrm{d} W(s) & \\ +\int_{0}^{t}\left\{\partial_{1} \mathfrak{A}_{0}(\mathfrak{X}(s-r), \mathfrak{X}(s)) \partial_{\mathfrak{X}} \mathfrak{X}(s-r)+\partial_{2} \mathfrak{A}_{0}(\mathfrak{X}(s-r), \mathfrak{X}(s)) \partial_{\mathfrak{X}} \mathfrak{X}(s)\right\} \mathrm{d} s \quad(0 \leq t \leq T) .\end{cases}
$$

Let $\mathfrak{U}=\{\mathfrak{U}(t) ;-r \leq t \leq T\}$ and $\hat{\mathfrak{U}}=\{\hat{\mathfrak{U}}(t) ;-r \leq t \leq T\}$ be $\mathbb{R}^{2}$-valued processes determined by the following ordinary differential equations:

$$
\begin{array}{lc}
\mathfrak{U}(t)=\hat{\mathfrak{U}}(t)=I_{2} & (-r \leq t \leq 0), \\
\mathfrak{U}(t)=I_{2}+\int_{0}^{t} \partial_{2} \mathfrak{A}_{0}(\mathfrak{X}(s-r), \mathfrak{X}(s)) \mathfrak{U}(s) \mathrm{d} s & (0 \leq t \leq T), \\
\hat{\mathfrak{U}}(t)=I_{2}-\int_{0}^{t} \hat{\mathfrak{U}}(s) \partial_{2} \mathfrak{A}_{0}(\mathfrak{X}(s-r), \mathfrak{X}(s)) \mathrm{d} s & (0 \leq t \leq T) .
\end{array}
$$

Then, it is clear that $\mathfrak{U}(t) \hat{\mathfrak{U}}(t)=\hat{\mathfrak{U}}(t) \mathfrak{U}(t)=I_{2}$, via the Itô formula.

Now, we shall consider the negative-order moment of $\operatorname{det} \mathfrak{V}(t)$. Moreover, for $0 \vee(t-r) \leq u \leq t$, we see that $\mathfrak{Z}(t, u)=\mathfrak{U}(t) \hat{\mathfrak{U}}(u)$ is invertible, because

$$
\begin{aligned}
\mathfrak{Z}(t, u)= & I_{2}+\int_{u}^{t} \partial_{1} \mathfrak{A}_{1}(\mathfrak{X}(s-r), \mathfrak{X}(s)) \mathfrak{Z}(s-r, u) \mathrm{d} W(s) \\
& +\int_{u}^{t}\left\{\partial_{1} \mathfrak{A}_{0}(\mathfrak{X}(s-r), \mathfrak{X}(s)) \mathfrak{Z}(s-r, u)+\partial_{2} \mathfrak{A}_{0}(\mathfrak{X}(s-r), \mathfrak{X}(s)) \mathfrak{Z}(s, u)\right\} \mathrm{d} s \\
= & I_{2}+\int_{u}^{t} \partial_{2} \mathfrak{A}_{0}(\mathfrak{X}(s-r), \mathfrak{X}(s)) \mathfrak{Z}(s, u) \mathrm{d} s
\end{aligned}
$$

for $0 \vee(t-r) \leq u \leq t$,

$$
\mathfrak{U}(t) \hat{\mathfrak{U}}(u)=I_{2}+\int_{u}^{t} \partial_{2} \mathfrak{A}_{0}(\mathfrak{X}(s-r), \mathfrak{X}(s)) \mathfrak{U}(s) \hat{\mathfrak{U}}(u) \mathrm{d} s,
$$

and the uniqueness of the solutions. Remark that

$$
\begin{aligned}
\mathfrak{V}(t) & \left.=\int_{0}^{t}\left(\mathfrak{Z}(t, u) \mathfrak{A}_{1}(\mathfrak{X}(u-r))\right)\left(\mathfrak{Z}(t, u) \mathfrak{A}_{1}(\mathfrak{X}(u-r))\right)\right)^{*} \mathrm{~d} u \\
& \geq \int_{0 \vee(t-r)}^{t}\left(\mathfrak{Z}(t, u) \mathfrak{A}_{1}(\mathfrak{X}(u-r))\right)\left(\mathfrak{Z}(t, u) \mathfrak{A}_{1}(\mathfrak{X}(u-r))\right)^{*} \mathrm{~d} u
\end{aligned}
$$




$$
\begin{aligned}
& =\mathfrak{U}(t)\left\{\int_{0 \vee(t-r)}^{t}\left(\hat{\mathfrak{U}}(u) \mathfrak{A}_{1}(\mathfrak{X}(u-r))\right)\left(\hat{\mathfrak{U}}(u) \mathfrak{A}_{1}(\mathfrak{X}(u-r))\right)^{*} \mathrm{~d} u\right\} \mathfrak{U}(t)^{*} \\
& =: \mathfrak{U}(t) \check{\mathfrak{V}}(t) \mathfrak{U}(t)^{*} .
\end{aligned}
$$

Here, the second inequality is in the matrix sense. It is clear that $\sup _{t \in[-r, T]}\|\mathfrak{U}(t)\|$ is in $\mathbb{L}^{p}(\Omega)$ for any $p>1$. Hence, we have to attack the negative-order moment of $\operatorname{det} \check{\mathfrak{V}}(t)$.

Remark that $\mathfrak{A}_{1}(\mathfrak{X}(u-r))$ is $\mathscr{F}_{0 \vee(t-r)}$-measurable for $0 \vee(t-r) \leq u \leq t$. When we study the lower estimate of $\breve{\mathfrak{V}}(t)$, we can regard the term $\mathfrak{A}_{1}(\mathfrak{X}(u-r))$ as the constant in the integrand of $\check{\mathfrak{V}}(t)$, by taking the conditional expectation on $\mathscr{F}_{0 \vee}(t-r)$. Let $\left(\alpha_{0}, \beta_{0}\right) \in \mathbb{R}^{2}$ be fixed. Since

$$
\mathfrak{A}_{1}\left(\alpha_{0}, \beta_{0}\right)=\left(\begin{array}{c}
\tilde{A}_{1}\left(\alpha_{0}\right) \\
0
\end{array}\right), \quad \mathfrak{A}_{0}\left(\left(\alpha_{0}, \beta_{0}\right),(\alpha, \beta)\right)=\left(\begin{array}{c}
-\tilde{A}_{1}\left(\alpha_{0}\right)^{2} / 2 \\
\exp (\alpha)
\end{array}\right)
$$

for $(\alpha, \beta) \in \mathbb{R}^{2}$, we see that

$$
\begin{aligned}
{\left[\mathfrak{A}_{0}, \mathfrak{A}_{1}\right]\left(\left(\alpha_{0}, \beta_{0}\right),(\alpha, \beta)\right) } & =-\partial_{(\alpha, \beta)} \mathfrak{A}_{0}\left(\left(\alpha_{0}, \beta_{0}\right),(\alpha, \beta)\right) \mathfrak{A}_{1}\left(\alpha_{0}, \beta_{0}\right) \\
& =\left(\begin{array}{c}
0 \\
-\tilde{A}_{1}\left(\alpha_{0}\right) \exp (\alpha)
\end{array}\right) .
\end{aligned}
$$

The dimension of the linear space generated by $\mathfrak{A}_{1}\left(\alpha_{0}, \beta_{0}\right)$ and $\left[\mathfrak{A}_{0}, \mathfrak{A}_{1}\right]\left(\left(\alpha_{0}, \beta_{0}\right),(\alpha, \beta)\right)$ is 2 for all $(\alpha, \beta) \in \mathbb{R}^{2}$, because of the uniformly elliptic condition (13) on $A_{1}$. Hence, we can conclude that the inverse of $\operatorname{det} \breve{\mathfrak{V}}(t)$ is in $\mathbb{L}^{p}(\Omega)$ for any $p>1$, which implies that the probability law of the $\mathbb{R}^{2}$-valued random variable $\mathfrak{X}(t)$ admits a smooth density with respect to the Lebesgue measure over $\mathbb{R}^{2}$. See [11] on details. Therefore, we can justify the existence of the smooth density for the probability law of the $\mathbb{R}$-valued random variable $Y(t)=\tilde{Y}(t) / t$ with respect to the Lebesgue measure over $\mathbb{R}$. The proof is complete.

Remark 5 Consider the case of $0 \leq t \leq r$. Then, we can derive

$$
\begin{aligned}
& \mathfrak{X}(t)=\left(\begin{array}{l}
\tilde{X}(t) \\
\tilde{Y}(t)
\end{array}\right)=\left(\begin{array}{c}
\tilde{x} \\
\tilde{y}
\end{array}\right)+\int_{0}^{t}\left(\begin{array}{c}
-\tilde{A}_{1}(\tilde{x})^{2} / 2 \\
\exp (\tilde{X}(s))
\end{array}\right) \mathrm{d} s+\int_{0}^{t}\left(\begin{array}{c}
\tilde{A}_{1}(\tilde{x}) \\
0
\end{array}\right) \mathrm{d} W(s), \\
& \partial_{\tilde{X}} \mathfrak{X}(t)(1,0)^{*}=\left(\begin{array}{c}
\partial_{\tilde{x}} \tilde{X}(t) \\
\partial_{\tilde{x}} \tilde{Y}(t)
\end{array}\right)=\left(\begin{array}{c}
1-\tilde{A}_{1}(\tilde{x}) \tilde{A}_{1}^{\prime}(\tilde{x}) t+\tilde{A}_{1}^{\prime}(\tilde{x}) W(t) \\
\int_{0}^{t}\left(1-\tilde{A}_{1}(\tilde{x}) \tilde{A}_{1}^{\prime}(\tilde{x}) s+\tilde{A}_{1}^{\prime}(\tilde{x}) W(s)\right) \exp (\tilde{X}(s)) \mathrm{d} s
\end{array}\right), \\
& \mathfrak{U}(t)=\left(\begin{array}{cc}
1 & 0 \\
\tilde{Y}(t) & 1
\end{array}\right), \quad \hat{\mathfrak{U}}(t)=\left(\begin{array}{cc}
1 & 0 \\
-\tilde{Y}(t) & 1
\end{array}\right), \quad \mathfrak{Z}(t, u)=\left(\begin{array}{cc}
1 & 0 \\
\tilde{Y}(t)-\tilde{Y}(u) & 1
\end{array}\right) \mathbb{I}_{(u \leq t)} .
\end{aligned}
$$

Since $\hat{\mathfrak{U}}(u) \mathfrak{A}_{1}(\mathfrak{X}(u-r))=\left(\tilde{A}_{1}(\tilde{x}),-\tilde{A}_{1}(\tilde{x}) \tilde{Y}(u)\right)^{*}$, we have

$$
\delta\left(\left(\hat{\mathfrak{U}}(\cdot) \mathfrak{A}_{1}(\mathfrak{X}(\cdot-r))\right)^{*}\right)=\int_{0}^{t}\left(\tilde{A}_{1}(\tilde{x}),-\tilde{A}_{1}(\tilde{x}) \tilde{Y}(u)\right) \mathrm{d} W(u) .
$$


Recall $\breve{\mathfrak{V}}(t)=\hat{\mathfrak{U}}(t) \mathfrak{V}(t) \hat{\mathfrak{U}}(t)^{*}$. Then, we see that

$$
\begin{aligned}
& \check{\mathfrak{V}}(t)=\left(\begin{array}{cc}
\tilde{A}_{1}(\tilde{x})^{2} t & -\tilde{A}_{1}(\tilde{x})^{2} \int_{0}^{t} \tilde{Y}(v) \mathrm{d} v \\
-\tilde{A}_{1}(\tilde{x})^{2} \int_{0}^{t} \tilde{Y}(v) \mathrm{d} v & \tilde{A}_{1}(\tilde{x})^{2} \int_{0}^{t} \tilde{Y}(v)^{2} \mathrm{~d} v
\end{array}\right) \\
& \check{\mathfrak{V}}(t)^{-1}=\frac{1}{\operatorname{det} \check{\mathfrak{V}}(t)}\left(\begin{array}{cc}
\tilde{A}_{1}(\tilde{x})^{2} \int_{0}^{t} \tilde{Y}(v)^{2} \mathrm{~d} v & \tilde{A}_{1}(\tilde{x})^{2} \int_{0}^{t} \tilde{Y}(v) \mathrm{d} v \\
\tilde{A}_{1}(\tilde{x})^{2} \int_{0}^{t} \tilde{Y}(v) \mathrm{d} v & \tilde{A}_{1}(\tilde{x})^{2} t
\end{array}\right)=: \frac{\Theta(t)}{\operatorname{det} \check{\mathfrak{V}}(t)} .
\end{aligned}
$$

Since the Malliavin derivatives of $\tilde{X}(t), \partial_{\tilde{X}} \tilde{X}(t), \tilde{Y}(t)$ and $\partial_{\tilde{x}} \tilde{Y}(t)$ can be computed as

$$
\begin{aligned}
& \frac{\mathrm{d}}{\mathrm{d} u} D_{u} \tilde{X}(t)=\tilde{A}_{1}(\tilde{x}) \mathbb{I}_{(u \leq t)}, \quad \frac{\mathrm{d}}{\mathrm{d} u} D_{u} \partial_{\tilde{x}} \tilde{X}(t)=\tilde{A}_{1}^{\prime}(\tilde{x}) \mathbb{I}_{(u \leq t)}, \\
& \frac{\mathrm{d}}{\mathrm{d} u} D_{u} \tilde{Y}(t)=\tilde{A}_{1}(\tilde{x})(\tilde{Y}(t)-\tilde{Y}(u)) \mathbb{I}_{(u \leq t)}, \\
& \frac{\mathrm{d}}{\mathrm{d} u} D_{u} \partial_{\tilde{x}} \tilde{Y}(t)=\tilde{A}_{1}^{\prime}(\tilde{x})(\tilde{Y}(t)-\tilde{Y}(u)) \mathbb{I}_{(u \leq t)}+\tilde{A}_{1}(\tilde{x})\left(\partial_{\tilde{x}} \tilde{Y}(t)-\partial_{\tilde{x}} \tilde{Y}(u)\right) \mathbb{I}_{(u \leq t)},
\end{aligned}
$$

we see that

$$
\begin{aligned}
& \frac{\mathrm{d}}{\mathrm{d} u} D_{u} \operatorname{det} \check{\mathfrak{V}}(t)=2 \tilde{A}_{1}(\tilde{x})^{5}\left(\int_{0}^{t} \int_{u}^{t}(\tilde{Y}(v)-\tilde{Y}(u))(\tilde{Y}(v)-\tilde{Y}(\sigma)) \mathrm{d} v \mathrm{~d} \sigma\right) \mathbb{I}_{(u \leq t)}, \\
& \frac{\mathrm{d}}{\mathrm{d} u} D_{u} \Theta(t)=\tilde{A}_{1}(\tilde{x})^{3}\left(\begin{array}{cc}
2 \int_{u}^{t} \tilde{Y}(v)(\tilde{Y}(v)-\tilde{Y}(u)) \mathrm{d} v & \int_{u}^{t}(\tilde{Y}(v)-\tilde{Y}(u)) \mathrm{d} v \\
\int_{u}^{t}(\tilde{Y}(v)-\tilde{Y}(u)) \mathrm{d} v & 0
\end{array}\right) \mathbb{I}_{(u \leq t)}, \\
& \frac{\mathrm{d}}{\mathrm{d} u} D_{u} \hat{\mathfrak{U}}(t)=\tilde{A}_{1}(\tilde{x})\left(\begin{array}{cc}
0 & 0 \\
-(\tilde{Y}(t)-\tilde{Y}(u)) & 0
\end{array}\right) \mathbb{I}_{(u \leq t)}, \\
& \frac{\mathrm{d}}{\mathrm{d} u} D_{u} \partial_{\tilde{X}} \mathfrak{X}(t)(1,0)^{*}=\partial_{\tilde{x}}\left(\begin{array}{c}
\tilde{A}_{1}(\tilde{x}) \\
\tilde{A}_{1}(\tilde{x})(\tilde{Y}(t)-\tilde{Y}(u))
\end{array}\right) \mathbb{I}_{(u \leq t)} .
\end{aligned}
$$

Write $\Delta(t):=t \int_{0}^{t} \tilde{Y}(v)^{2} \mathrm{~d} v-\left(\int_{0}^{t} \tilde{Y}(v) \mathrm{d} v\right)^{2}$. Since $\mathfrak{Z}(t, u)=\mathfrak{U}(t) \hat{\mathfrak{U}}(u)$ for $0 \leq t \leq r$, Proposition 1.3.5 (p.40) in [16] enables us to obtain

$$
\begin{aligned}
\delta( & \left(\left(\mathfrak{Z}(t, \cdot) \mathfrak{A}_{1}(\mathfrak{X}(\cdot-r))\right)^{*} \mathfrak{V}(t)^{-1} \partial_{\tilde{\mathfrak{X}}} \mathfrak{X}(t)\right)(1,0)^{*} \\
= & \delta\left(\left(\hat{\mathfrak{U}}(\cdot) \mathfrak{A}_{1}(\mathfrak{X}(\cdot-r))\right)^{*}\right) \frac{\Theta(t)}{\operatorname{det} \check{\mathfrak{V}}(t)} \hat{\mathfrak{U}}(t) \partial_{\tilde{\mathfrak{X}}} \mathfrak{X}(t)(1,0)^{*} \\
& -\int_{0}^{t}\left(\hat{\mathfrak{U}}(u) \mathfrak{A}_{1}(\mathfrak{X}(u-r))\right)^{*} \frac{\mathrm{d}}{\mathrm{d} u} D_{u}\left(\frac{\Theta(t)}{\operatorname{det} \check{\mathfrak{V}}(t)} \hat{\mathfrak{U}}(t) \partial_{\tilde{\mathfrak{X}}} \mathfrak{X}(t)(1,0)^{*}\right) \mathrm{d} u \\
= & \left(\int_{0}^{t}\left(\tilde{A}_{1}(\tilde{x}),-\tilde{A}_{1}(\tilde{x}) \tilde{Y}(u)\right) \mathrm{d} W(u)\right) \frac{\Theta(t)}{\operatorname{det} \check{\mathfrak{V}}(t)} \hat{\mathfrak{U}}(t) \partial_{\tilde{\mathfrak{X}}} \mathfrak{X}(t)(1,0)^{*} \\
& +\int_{0}^{t}\left(\tilde{A}_{1}(\tilde{x}),-\tilde{A}_{1}(\tilde{x}) \tilde{Y}(u)\right) \frac{\frac{\mathrm{d}}{\mathrm{d} u} D_{u} \operatorname{det} \check{\mathfrak{V}}(t)}{\operatorname{det} \check{\mathfrak{V}}(t)^{2}} \Theta(t) \hat{\mathfrak{U}}(t) \partial_{\tilde{\mathfrak{X}}} \mathfrak{X}(t)(1,0)^{*} \mathrm{~d} u
\end{aligned}
$$




$$
\begin{aligned}
& -\int_{0}^{t}\left(\tilde{A}_{1}(\tilde{x}),-\tilde{A}_{1}(\tilde{x}) \tilde{Y}(u)\right) \frac{1}{\operatorname{det} \check{\mathfrak{V}}(t)}\left(\frac{\mathrm{d}}{\mathrm{d} u} D_{u} \Theta(t)\right) \hat{\mathfrak{U}}(t) \partial_{\tilde{\mathfrak{X}}} \mathfrak{X}(t)(1,0)^{*} \mathrm{~d} u \\
& -\int_{0}^{t}\left(\tilde{A}_{1}(\tilde{x}),-\tilde{A}_{1}(\tilde{x}) \tilde{Y}(u)\right) \frac{\Theta(t)}{\operatorname{det} \check{\mathfrak{V}}(t)}\left(\frac{\mathrm{d}}{\mathrm{d} u} D_{u} \hat{\mathfrak{U}}(t)\right) \partial_{\tilde{\mathfrak{X}}} \mathfrak{X}(t)(1,0)^{*} \mathrm{~d} u \\
& -\int_{0}^{t}\left(\tilde{A}_{1}(\tilde{x}),-\tilde{A}_{1}(\tilde{x}) \tilde{Y}(u)\right) \frac{\Theta(t)}{\operatorname{det} \check{\mathfrak{V}}(t)} \hat{\mathfrak{U}}(t)\left(\frac{\mathrm{d}}{\mathrm{d} u} D_{u} \partial_{\tilde{\mathfrak{X}}} \mathfrak{X}(t)(1,0)^{*}\right) \mathrm{d} u \\
& =\left(\int_{0}^{t}(1,-\tilde{Y}(u)) \mathrm{d} W(u)\right) \frac{\Theta(t)}{\tilde{A}_{1}(\tilde{x})^{3} \Delta(t)} \check{\mathfrak{U}}(t)\left(\begin{array}{c}
\partial_{\tilde{x}} \tilde{X}(t) \\
\partial_{\tilde{x}} \tilde{Y}(t)
\end{array}\right) \\
& +\int_{0}^{t}(1,-\tilde{Y}(u)) \frac{2 \int_{0}^{t} \int_{u}^{t}(\tilde{Y}(v)-\tilde{Y}(u))(\tilde{Y}(v)-\tilde{Y}(\sigma)) \mathrm{d} v \mathrm{~d} \sigma}{\tilde{A}_{1}(\tilde{x})^{2} \Delta(t)^{2}} \mathrm{~d} u \Theta(t) \check{\mathfrak{U}}(t)\left(\begin{array}{l}
\partial_{\tilde{x}} \tilde{X}(t) \\
\partial_{\tilde{X}} \tilde{Y}(t)
\end{array}\right) \\
& -\int_{0}^{t}(1,-\tilde{Y}(u)) \frac{1}{\Delta(t)}\left(\begin{array}{cc}
\int_{u}^{t} 2 \tilde{Y}(v)(\tilde{Y}(v)-\tilde{Y}(u)) \mathrm{d} v & \int_{u}^{t}(\tilde{Y}(v)-\tilde{Y}(u)) \mathrm{d} v \\
\int_{u}^{t}(\tilde{Y}(v)-\tilde{Y}(u)) \mathrm{d} v & 0
\end{array}\right) \mathrm{d} u \tilde{U}(t)\left(\begin{array}{c}
\partial_{\tilde{x}} \tilde{X}(t) \\
\partial_{\tilde{x}} \tilde{Y}(t)
\end{array}\right) \\
& -\int_{0}^{t}(1,-\tilde{Y}(u)) \frac{\Theta(t)}{\tilde{A}_{1}(\tilde{x})^{2} \Delta(t)}\left(\begin{array}{cc}
0 & 0 \\
-(\tilde{Y}(t)-\tilde{Y}(u)) & 0
\end{array}\right) \mathrm{d} u\left(\begin{array}{l}
\partial_{\tilde{x}} \tilde{X}(t) \\
\partial_{\tilde{x}} \tilde{Y}(t)
\end{array}\right) \\
& -\int_{0}^{t}(1,-\tilde{Y}(u)) \frac{\Theta(t)}{\tilde{A}_{1}(\tilde{x})^{3} \Delta(t)} \check{\mathfrak{U}}(t) \partial_{\tilde{x}}\left(\begin{array}{c}
\tilde{A}_{1}(\tilde{x}) \\
\tilde{A}_{1}(\tilde{x})(\tilde{Y}(t)-\tilde{Y}(u))
\end{array}\right) \mathrm{d} u .
\end{aligned}
$$

Remark 6 Consider the case $A_{1}(z)=\alpha_{1}$ and $\tilde{y}=0$, where $\alpha_{1}$ is a constant. Since the equations (21) and (22) are

$$
\tilde{X}(t)=\tilde{x}-\frac{\alpha_{1}^{2}}{2} t+\alpha_{1} W(t), \quad \tilde{Y}(t)=\int_{0}^{t} \exp (\tilde{X}(s)) \mathrm{d} s
$$

for $t \in[0, T]$, our situation here is the Asian-type option for the classical Black-Scholes model under a risk-neutral measure dP. As seen in Remark 5, we can also compute

$$
\begin{aligned}
\delta & \left(\left(\mathfrak{Z}(t, \cdot) \mathfrak{A}_{1}\right)^{*} \mathfrak{V}(t)^{-1} \partial_{\tilde{X}} \mathfrak{X}(t)\right)(1,0)^{*} \\
= & \frac{1}{\alpha_{1} \Delta(t)}\left(W(t),-\int_{0}^{t} \tilde{Y}(u) \mathrm{d} W(u)\right)\left(\begin{array}{c}
\int_{0}^{t} \tilde{Y}(v)^{2} \mathrm{~d} v \\
\int_{0}^{t} \tilde{Y}(v) \mathrm{d} v
\end{array}\right) \\
& +\frac{2}{\Delta(t)^{2}} \int_{0}^{t}(1,-\tilde{Y}(u))\left(\int_{0}^{t} \int_{u}^{t}(\tilde{Y}(v)-\tilde{Y}(u))(\tilde{Y}(v)-\tilde{Y}(\sigma)) \mathrm{d} v \mathrm{~d} \sigma\right) \mathrm{d} u\left(\begin{array}{l}
\left.\int_{0}^{t} \tilde{Y}(v)^{2} \mathrm{~d} v\right) \\
\int_{0}^{t} \tilde{Y}(v) \mathrm{d} v
\end{array}\right) \\
& -\frac{1}{\Delta(t)} \int_{0}^{t}(1,-\tilde{Y}(u))\left(\begin{array}{c}
\int_{u}^{t} 2 \tilde{Y}(v)(\tilde{Y}(v)-\tilde{Y}(u)) \mathrm{d} v \\
\int_{u}^{t}(\tilde{Y}(v)-\tilde{Y}(u)) \mathrm{d} v
\end{array}\right) \mathrm{d} u .
\end{aligned}
$$


Remark 7 Let $0<r_{0} \leq r$ be a constant, and denote by $\Pi: C([-r, 0] ; \mathbb{R}) \rightarrow C\left(\left[-r,-r_{0}\right] ; \mathbb{R}\right)$ the projection such that $\Pi(f)=\left\{f(s) ;-r \leq s \leq-r_{0}\right\}$ for $f \in C([-r, 0] ; \mathbb{R})$. Similarly to the studies stated above, we can also discuss the case where the process $X=\{X(t) ;-r \leq t \leq T\}$ is determined by the equation:

$$
X(t)= \begin{cases}x & (-r \leq t \leq 0) \\ x+\int_{0}^{t} A_{0}\left(\Pi\left(X_{s}\right)\right) X(s) \mathrm{d} s+\int_{0}^{t} A_{1}\left(\Pi\left(X_{s}\right)\right) X(s) \mathrm{d} W(s) & (0<t \leq T),\end{cases}
$$

where $A_{i} \in C_{0+, b}^{1}\left(C\left(\left[-r,-r_{0}\right] ; \mathbb{R}\right) ; \mathbb{R}\right)(i=0,1)$ with the uniformly elliptic condition on $A_{1}$ :

$$
\inf _{f \in C([-r, 0] ; \mathbb{R})}\left(A_{1}(\Pi(f))\right)^{2} \geq C_{13} .
$$

\section{Acknowledgements}

This work is supported by JSPS KAKENHI Grant Number 23740083 (Grant-in-Aid for Young Scientists (B)). This work was motivated by a private discussion with Alexey Kulik during his stay in Japan on February in 2015. The author is very grateful to him for his valuable comments and suggestions.

\section{References}

[1] M. Arriojas, Y. Hu, S.-E. A. Mohammed, G. Pap: A delayed Black and Scholes formula, Stochastic Analysis and Applications, 25, no. 2, (2007), pp. 471-492.

[2] D. R. Bell and S.-E. A. Mohammed: The Malliavin calculus and stochastic delay equations, Journal of Functional Analysis, 99, no. 1, (1991), pp. 75-99.

[3] D. R. Bell and S.-E. A. Mohammed: Smooth densities for degenerate stochastic delay equations with hereditary drift, The Annals of Probability, 23, no. 4, (1995), pp. 1875-1894.

[4] M.-H. Chang, T. Pang and M. Pemy: An approximation scheme for Black-Scholes equations with delays, Journal of Systems Science and Complexity, 23, no. 3, (2010), pp.438 - 455.

[5] M.-H. Chang and R. Youree: The European option with hereditary price structures: Basic theory, Applied Mathematics and Computation, 102, (1999), pp. 279 - 296.

[6] M.-H. Chang and R. Youree: Infinite-dimensional Black-Scholes with hereditary structure, Applied Mathematics and Optimization, 56, (2007), pp.395 - 424.

[7] N. Ikeda and S. Watanabe: Stochastic differential equations and diffusion processes, 2nd edition, North-Holland/Kodansha, Tokyo 1989. 
[8] K. Ito and M. Nisio: On stationary solutions of a stochastic differential equation, Journal of Mathematics of Kyoto University, 4, no. 1, (1964), pp.1-75.

[9] R. Kawai and A. Takeuchi: Greeks formulas for an asset price model with gamma processes, Mathematical Finance 21 (2011), no. 4, 723-742.

[10] A. Kitagawa and A. Takeuchi: Asymptotic behavior of densities for stochastic functional differential equations, International Journal of Stochastic Analysis, 2013 (2013), pp. 1-17.

[11] T. Komatsu and A. Takeuchi: Simplified probabilistic approach to the Hörmander theorem, Osaka Journal of Mathematics, 38, no. 3, (2001), pp.681-691.

[12] S. Kusuoka and D. W. Stroock: Applications of the Malliavin calculus. I, in Stochastic Analysis (Katata/Kyoto, 1982), ed. K. Ito, pp.271-306, North-Holland, Amsterdam, 1984.

[13] N. McWilliams and S. Sabanis: Arithmetic Asian options under stochastic delay models, Applied Mathematical Finance, 18, no. 5, (2011), pp. 423-446.

[14] S.-E. A. Mohammed: Stochastic functional differential equations, Pitman, Boston, MA, USA, 1984.

[15] S.-E. A. Mohammed: Stochastic differential systems with memory: theory, examples and applications, in Stochastic Analysis and Related Topics VI, (Geilo, 1996), eds. L. Decreusefond, J. Gjerde, B. Oksendal and A.S. Ustunel, vol. 42 of Progress in Probability, pp.1-77, Birkhäuser, Boston, MA, USA, 1998.

[16] D. Nualart: The Malliavin calculus and related topics, Springer-Verlag, 2nd edition, Berlin, 2006.

[17] A. Takeuchi: Bismut-Elworthy-Li type formulae for stochastic differential equations with jumps, Journal of Theoretical Probability 23, no. 2, (2010), 576-604. 